\title{
Enrichment-Free Identification of Native Definitive (EnFIND) O-glycoproteome of antibodies in autoimmune diseases
}

\author{
Xue Sun ${ }^{1 \#}$, Jianhui Cheng ${ }^{2 \#}$, Wenmin Tian ${ }^{1,3 \#}$, Shuaixin Gao ${ }^{1}$, Jiangtao $\mathrm{Guo}^{1}$, Fanlei Hu${ }^{5,6}$, \\ Hong Zhang ${ }^{4}$, Xiaojun Huang ${ }^{7,8}$, Da Yong Chen ${ }^{3}$, Yang Chen ${ }^{1,3^{*}}$, Catherine CL Wong ${ }^{1,3,4,6,8^{*}}$
}

${ }^{1}$ Center for Precision Medicine Multi-Omics Research, Peking University Health Science Center, Peking University, Beijing 100191, China.

${ }^{2}$ Department of Chemistry, University of British Columbia, Vancouver, BC, Canada.

${ }^{3}$ School of Basic Medical Sciences, Peking University Health Science Center, Beijing 100191, China.

${ }^{4}$ Peking University First Hospital, Beijing 100034, China.

${ }^{5}$ Department of Rheumatology and Immunology, Peking University People's Hospital \& Beijing Key Laboratory for Rheumatism Mechanism and Immune Diagnosis (BZ0135), Beijing, China.

${ }^{6}$ State Key Laboratory of Natural and Biomimetic Drugs, School of Pharmaceutical Sciences, Peking University, Beijing, China.

${ }^{7}$ Peking University People's Hospital, Peking University Institute of Hematology, Beijing Key Laboratory of Hematopoietic Stem Cell Transplantation, National Clinical Research Center for Hematologic Disease, No. 11 South Street of Xizhimen, Xicheng District, Beijing 100044, China.

${ }^{8}$ Peking-Tsinghua Center for Life Sciences, Beijing 100871, China

\# These authors contributed equally to this work

*Correspondence to:

chenyang1816185048@bjmu.edu.cn

catherine_wong@bjmu.edu.cn

Running title: No enrich O-glycoproteome identification 


\begin{abstract}
:
The detection of O-glycosylation at the proteome level has long been a challenging task and a roadblock for O-linked protein glycosylation research. We report an Enrichment-Free Identification of Native Definitive (EnFIND) O-glycoproteome using Trapped Ion Mobility Spectrometry coupled to TOF Mass Spectrometry (TIMS-TOF MS) for direct analysis of protein O-glycosylation in native samples with minimum sample requirement. This approach enabled separation of O-glycopeptide isomers, resolution of O-glycosites and O-glycoform, reduction of sample complexity, and increased sensitivity, thus greatly enhancing analysis of the O-glycoproteome of cell lysates, human serum and exosomes. In addition, we found that antibodies in human serum are highly O-glycosylated on variable, especially hypervariable regions and constant regions, which significantly increases antibody diversity. This method was used to successfully identify characteristic O-glycosylation features of autoimmune diseases.
\end{abstract}

Keywords: Antibody / Autoimmune disease / Enrichment -free / O-glycoproteome

\title{
Introduction:
}

O-glycosylation is one of the most abundant and diverse type of protein glycosylation. It mainly occurs on serine $(\mathrm{S})$, threonine $(\mathrm{T})$ or tyrosine $(\mathrm{Y})$ residues and initiates with the addition of GlcNAc, GalNAc, Fucose, Glucose, Galactose or Mannose. The initial monosaccharide can be further elongated to form large and complex glycan structures. The O-glycosylation at specific sites contribute to the diverse regulation of protein activities and serve a variety of biological functions(Marino et al, 2010). More importantly, Oglycosylation is related to diseases including congenital disorders, cancer and autoimmune diseases(Reily et al, 2019). Analysis of O-glycosylation at the proteome level has been a challenging task for decades. O-glycopeptide ions are highly heterogeneous and their signals 
are suppressed in mass spectrometry by unmodified peptides due to their low abundance. Identification of O-glycopeptides by mass spectrometry is further complicated by the lack of glycan consensus structures and the existence of glycoform isomers. Substantial improvements have been made for large-scale O-glycoproteomics analysis in complex biological samples(King et al, 2017; Mao et al, 2019; Schjoldager et al, 2012; Vakhrushev et al, 2013; Yang et al, 2018; Yang et al, 2014). For example, Vakhrushev’s group recently applied data-independent acquisition (DIA) for direct analysis of O-glycoproteins, which was a major advance in characterizing glycosites and glycans(Ye et al, 2019). However, these methods are all based on enrichment of certain types of glycans, mainly GalNAc-type glycans. Therefore, only GalNAc-type O-glycosylation has been heavily studied. A nonbiased comprehensive profile of glycan structures and O-glycosites at the whole proteome level has still not been satisfactorily achieved. High-resolution mass spectrometry-based proteomics has become the most powerful tool to study protein glycosylation. Sensitivity, sequencing speed and peak capacity are the key factors for achieving in-depth identification of the glycoproteome. The newly emerging trapped ion mobility spectrometry (TIMS) coupled with TOF mass spectrometry (TIMS-TOF) uses a revolutionary data acquisition technology called Parallel Accumulation - Serial Fragmentation (PASEF) which increases the sampling duty cycle to $100 \%$. This scanning speed, together with the capability to separate isomeric peptides, pushes the instrument sensitivity to a new height and thus makes it possible to analyze the glycoproteome at a much deeper level(Meier et al, 2015).

\section{Results:}

\section{Enrichment-Free Identification of Native Definitive (EnFIND) O-glycoproteome}

Taking advantage of the high sensitivity and high isomer separation capability of TIMS-TOF with PASEF, we herein developed an enrichment-free method to identify the native definitive 
(EnFIND) O-glycoproteome. We applied EnFIND to a whole cell lysate of A549 and human serum. To further challenge the sensitivity of this method, we also applied it to exosomes derived from cells or serum, which can only be purified in very limited amounts.

The workflow of EnFIND is shown in Figure 1A. A small amount (200 ng) of each sample was loaded directly into a nanoLC-TIMS-TOF after a standard pre-treatment process with dual-enzyme digestion to increase the peptide sequence coverage. The general mode of TIMS operation included two steps with ion accumulation (TIMS 1) and serial elution (TIMS 2) of ions separated on the basis of ion mobility from the TIMS device by decreasing the electrical field. The heatmap shows the nested $\mathrm{m} / \mathrm{z}$ and ion mobility distribution in the PASEF scan, which, together with retention time (RT), provides 3-dimensional (3-D) peptide information for the database search. The 3-D data and the 100\% duty cycle of PASEF are the two unique features that bring the sensitivity and thus the protein identification to a new level.

We used the total numbers of identified proteins (IDs) as a benchmark for the quality control of EnFIND (Fig 1B). The total 7149 and 943 protein IDs were identified in the A549 whole cell lysate and human serum, respectively. Under these conditions, we identified a total of 1738 and 350 O-glycoproteins in A549 whole cell lysate and human serum, respectively (Fig 1B). In combination of all samples analyzed, we identified a total of 2462 O-glycoproteins compared to the 2102 O-glycoproteins from recently published Glyco-DIA library. Among 2462 O-glycoproteins, 1886 O-glycoproteins specifically in this study (Fig 1C)(Ye et al, 2019). Moreover, 148 and 431 O-glycoproteins were identified exclusively in exosomes derived from A549 or serum (Fig EV1, EV2). This substantially increases the existing knowledge of the O-glycoproteome. Gene ontology (GO) analysis of the identified 
glycoproteins in cells and serum showed that various localizations, activities and functionalities are associated with O-glycoproteins, consistent with their important role in different aspects of biology (Fig EV3). The distribution of O-glycosites is consistent with previous reports, with the O-linkages occurring more frequently on serine (49.8\%) and threonine (37.6\%) than on tyrosine (12.6\%) (Fig 1D).

The high sensitivity of TIMS-TOF with PASEF is one of the key aspects of the EnFIND. Oglycoproteome and total peptides of O-glycoproteome share the same trend of distribution at $1 \mathrm{e} 3$ to $1 \mathrm{e} 5$ and $1 \mathrm{e} 3$ to $5 \mathrm{e} 4$, respectively (Fig $1 \mathrm{E}$, left and middle panels). However, Oglycopeptides show a different distribution pattern, with the relative density peaks shifted to $1 \mathrm{e} 2$ to $5 \mathrm{e} 3$, and only one peak at intensity $1 \mathrm{e} 3$ (Fig 1E, right panel). This demonstrates a successful coverage of low-abundance O-glycopeptides by TIMS-TOF with PASEF. Our data suggest that EnFIND delivers deep coverage of complex native samples.

In the EnFIND approach, we optimized the glycoform database search by constructing 14 types of O-glycoforms derived from the 6 monosaccharides based on previous literatures suggested high structural heterogeneity (Fig 1F)(Joshi et al, 2018; Sheikh et al, 2017). We found that GalNAc/GlcNAc $(203.08 \mathrm{~m} / \mathrm{z})$ O-glycosylation is indeed a major type, but other types of O-glycosylation are also abundant (Fig 1G).

\section{Features of the EnFIND method}

The ion suppression of very low-abundant O-glycosylated peptides by unmodified peptides is a major roadblock in the identification of O-glycoproteins. In the EnFIND method, we leveraged the capability of separation of isomers by ion mobility spectrometry to achieve full resolution of co-eluted O-glycopeptides and the corresponding unmodified peptide. The 
extracted ion chromatogram in Figure 2A shows that the O-linked GalNAc/GlcNAc peptide DSKPDTtAPPSSPK (precursor $m / z 815.895 z=+2$ ) and its unmodified form DSKPDTTAPPSSPK (precursor $m / z 714.35 z=+2$ ) cannot be separated by regular reversedphase chromatography, but they are well distinguished on the basis of ion mobility, presented in extracted ion mobilogram (EIM).

Identification of isoforms is always a "tough spot" for modern mass spectrometry. Most research groups focus on site-specific glycosylation, assisted by the different specific fragmentation features of modern mass spectrometry techniques, such as collisional induced dissociation (CID), electron transfer dissociation (ETD) and/or electron captured dissociation (ECD)(Darula et al, 2016; Halim et al, 2012; Hoffmann et al, 2016; Levery et al, 2015; Nilsson et al, 2009). In the EnFIND approach, TIMS-TOF with PASEF demonstrates the power of ion mobility spectrometry to identify isomeric O-glycopeptides. We distinguish two types of isomeric peptides in O-glycosylation: adjacent sites modified with the same glycoform and isomeric glycoforms at the same site. The two EIM peaks of DSKPDTtAPPSSPK (precursor $m / z 815.895 z=+2$ ) in Figure 2A indicate two isomeric glycoforms of GlcNAc and GalNAc, with mass of 203.08, added to the same threonine residue $(\mathrm{T})$. Co-eluting isomeric O-linked fucose (mass $=146.06)$ peptides of NDItAVTKEGK and NDITAVtKEGK show a total separation in ion mobility, resulting in high-quality MS2 spectra that enabled accurate localization of the two different threonine (T) sites (Fig 2B). More excitingly, we are able to identify complex glycan isomers. Figure 2C shows an MS2 spectrum of the doubly charged precursor peptide of DYYCLsG (precursor $m / z$ 694.77) with an O-linked trisaccharide glycan (mass $=511.19)$ modified at residue of serine (S) (Fig 2C, panel a). The EIC shows only one peak for the precursor, however, a full separation of the isomers of this trisaccharide glycan was visible in the EIM (peaks 1, 2, and 
3) (Fig 2C panel b, c). This trisaccharide glycan was composed of three monosaccharides, with mass of 146.06, 162.05 and 203.08 respectively. From the characteristic fragments identified in the MS2 spectra, we are able to deduce the most likely glycan structures corresponding to each peak (Fig 2C, panels d-f). The proposed glycan isoforms are listed in Figure 2D. The electron transfer diagrams show the most favorable trajectory of all fragments to each isoform, thus further supporting the glycan structures. Although we still cannot distinguish the monosaccharide Glc, Gal and Man (mass =162.05) or GlcNAc and GalNAc (mass $=203.08)$ in this trisaccharide glycan, our method achieves the highest resolution in isomer analysis in proteomics studies. The further confirmation of glycan structures will require release of glycans from purified glycoproteins followed by mass spectrum analysis and more information on chemical biology and glycosidic bond dissociation energy(Chai et al, 2018; Tang et al, 2018; Yu et al, 2012).

Antibodies are highly glycosylated proteins. Glycosylation on the variable and constant regions of antibodies is known to influence antigen binding, stability and effector functions, which are related to a variety of diseases(Arnold et al, 2007; Reily et al, 2019). Compared to $\mathrm{N}$-glycosylation, antibody O-glycosylation, especially on the proteomic level, has received less attention due to the lack of detection methods(de Haan et al, 2020). The sensitivity advantage of EnFIND prompted us to investigate the O-glycosylation of antibodies in human serum.

\section{Identification of O-glycosylation on antibodies}

We analyzed the O-glycosylation of antibodies in whole human serum, including total serum, high abundant proteins fraction, exosome fraction, and antibodies purified by protein A/G. Combining the data, we found that antibodies are highly O-glycosylated on all regions (Fig 
3A). 110 out of 144 total antibodies are O-glycosylated with 701 glycosites identified on both variable and constant region on heavy and light chain of antibodies with multiple glycoforms (Fig 3B, Table EV1). We identified O-glycosylation of all five isotypes, $\operatorname{IgG}, \operatorname{IgA}, \operatorname{IgM}, \operatorname{IgE}$ and IgD (Fig 3C, Table EV2). O-glycosylation of IgD and IgE are rarely reported, consistent with the low amount of $\operatorname{IgD}$ and $\operatorname{IgE}$ in human serum. The complementarity determining region (CDR), also named hypervariable region, of an antibody determines the antigen binding affinity and specificity. From the IMGT antibody database, we found that the relative frequencies of serine, threonine and tyrosine are high in CDR1 and CDR2 (Fig EV4). We detected 49 antibodies with O-glycosylation in CDR. Furthermore, 100 O-glycosites were identified including 38 isomeric glycopeptides caused by the same modifications on adjacent sites, and 28 glycoform isomers (Fig 3D). The MS2 spectrum of each identified isomeric glycopeptides was manually confirmed (Fig EV5). The heterogeneity of O-glycosylation on CDRs may further add to the diversity of the antibody repertoire.

We found that antibody was O-glycosylated at multiple sites and with different glycoforms. Thus, we hypothesized that antibody O-glycosylation raise antibody diversity in addition to amino acid sequence. We build a comprehensive map of antibody O-glycosylation in human serum from two healthy controls using EnFIND approach. The average number of Oglycosites or O-glycosites and O-glycoforms reached a plateau after 13 repeat runs (Fig 3E, F), with an average of 231 and 496 respectively in the two samples tested. What intrigued us is that the total antibodies ID identified in the two healthy controls are quite similar, with 81.8\% overlap (Fig 3G). Surprisingly, the O-glycosites and the O-glycosites with different glycoforms overlap by only $35.1 \%$ and $29.3 \%$, respectively (Fig 3H, I). These data demonstrated that antibody $\mathrm{O}$-glycosylation in the serum are highly diverse. The significance of this is currently a mystery. 


\section{Antibody O-glycosylation is correlated with autoimmune diseases}

Alterations of antibody glycosites or glycoforms can contribute to immune dysregulation and a range of autoimmune and chronic inflammatory diseases. However, current studies focus on antibody $\mathrm{N}$-glycosylation, and the O-glycosylation status is rarely determined(Reily et al, 2019). The EnFIND approach detect the O-glycosylation level of proteins at the native state. We next explored antibody O-glycosylation in diseases, with the aim of identifying diseaseassociated O-glycosylation patterns. We applied EnFIND to study the autoimmune diseases. Using the workflow described above (Fig 1A), we first selected the constantly identified Oglycosites. Then, we obtained the O-glycosylation level by calculating the ratio of total peptide-spectrum matches (PSM) of O-glycopeptide to the total PSM of the corresponding peptide. We analyzed 10 systemic lupus erythematosus (SLE) serum samples and 10 healthy controls (Table EV3). This allowed us to identify statistically different O-glycosylations on 8 O-glycosites in SLE which are located at hinge region of IGG1 (T225, T227), CH2 region of IGG1 (S241,T252), CH3 region of IGHG2 (S257), and constant regions of light chain IGLC7, IGLC3 and IGKC (Fig 4A, Fig EV6, Table EV4). Interestingly, O-glycosylation on S133 of the IGG1 CH1 region, S59 of IGLC3, and S14 and S20 of IGKC are significantly different between the autoimmune diseases rheumatoid arthritis (RA) and sjogren's syndrome (SS) (Fig 4C, Table EV4). We also studied plasma samples from patients with mild and critical IgA nephropathy, with the aim of finding clues about the transition from the mild cases to critical cases. Plasma samples were analyzed from 8 mild IgA nephropathy cases, 8 critical cases and 10 healthy controls. We found statistically different O-glycosylations on 8 O-glycosites for IgA nephropathy. They are located on the hinge region of IGG1 (S221, T225, T227, S241) and CH2 region of IGG1 (T252, T262), and the light chain constant region of IGLC3 (Fig 4B, Fig EV5, Table EV4). Glycosylation on the antibody light chain has rarely been reported before, but we consistently identified altered O-glycosylation of the antibody 
light chain in autoimmune diseases. Altered O-glycosylation in the IgA1 hinge region is reported to be related to IgA nephropathy(de Haan et al, 2020). However, our EnFIND approach did not confidently identify the IgA1 hinge region. This might due to the lack of trypsin and AspN cleavage sites in the IgA1 hinge region. We identified that T361 on IGG1, S46 on IGLC3 and T90 on IGLC7 distinguished the mild cases from the critical cases (Fig 4D, Table EV4). It is worth noting that most studies on O-glycosylation mainly focused on Mucin-type (GalNAc-type) O-glycosylation. We identified disease specific O-glycosylation with a variety of O-glycoforms including O-Fucose and O-Glc/Gal/Man as well as disaccharide $308.11(\mathrm{~m} / \mathrm{z})$, composed of Glc/Gal/Man $(162.05 \mathrm{~m} / \mathrm{z})$ and Fucose $(146.06 \mathrm{~m} / \mathrm{z})$. Thus, identification of other types of O-glycosylation other than GalNAc-type will be advantageous for disease-related O-glycosylation studies.

\section{Discussion:}

We developed an enrichment-free method to identify the native definitive (EnFIND) Oglycoproteome, which enabled us to simultaneously profile the O-glycosites and Oglycoforms from complex native samples. The core advantage of this method relies on the additional separation of isomers on the basis of ion mobility, which greatly decreases the complexity of the sample and further increases the sensitivity. EnFIND requires only trace amounts of sample, making the method suitable for analysis of a wide variety of samples. Without enrichment process, the EnFIND method is detecting the native state, which might reflect the true in vivo status of protein O-glycosylation in biological systems, such as whole cell lysates, cell-derived exosomes, serum and exosomes from serum. The 14 types of Oglycoforms included in the data processing greatly increase the number of identified $\mathrm{O}$ glycosites as well as the number of O-glycoforms on one site. Here, we have provided solid evidence that EnFIND is highly effective in separating modified and unmodified peptides and O-glycopeptides isomers, and it can also differentiate isomeric glycoforms. The advantages 
of EnFIND will guarantee its wide application in O-glycosylation research. Specifically, it will greatly improve O-glyco-library construction for the recently published DIA method for O-glycosylation analysis(Ye et al, 2019).

We applied EnFIND to the analysis of serum samples, and we report for the first time the large-scale O-glycosylation profiling of serum antibodies. We found that antibodies were Oglycosylated in the variable, constant and hinge regions. Most interestingly, we identified a number of O-glycosites located on the CDRs. CDRs play critical roles in epitope recognition and the subsequent immune response, especially CDR3 of the heavy chain, which lies at the center of the antigen-binding site(Vale et al, 2015). Since the sequence of CDR3 is largely missing in database of protein sequence, we only identified a few glycosites on CDR3, but we hypothesize that there should be more O-glycosylation events in this region. So far, we have no evidence of how O-glycosylation on CDRs regulates antibody activity; however, we can speculate that these modifications may regulate antigen binding affinity, antigen-antibody recognition and antibody stability. Different O-glycosites and different types of Oglycoforms, may further increase antibody diversity in addition to primary amino acid sequence. Most notably, antibody O-glycosylation patterns determined a personalized condition among people, the significance of which remains to be determined. The EnFIND method also enables us to investigate the changes in O-glycosylation in diseases. The identified disease-differential O-glycosites provide clues about disease mechanisms. However, the molecular mechanisms underlying the differences in antibody O-glycosylation in autoimmune diseases require extensive studies. 
We are now at the initial stage of O-glycoproteome study and there is far more to be explored, especially the functions of O-glycosylation on antibodies. The EnFIND method will benefit biomedical, biopharmaceutical and clinical research. Furthermore, antibodies account for only a small proportion of total O-glycosylated proteins. EnFIND will lead us to a broad identification of protein O-glycosylation in cell, tissue and serum samples to understand how O-glycosylation functions and will boost the knowledge of protein post translational modifications. It will also lead to the discovery of characteristic O-glyco-markers for diseases, which may open up a novel and exciting field for future study.

\section{Materials and Methods :}

\section{Chemicals and reagents:}

Trichloroacetic acid (TCA), Urea (ACS reagent, 99.0-100.5 \%), Iodoacetamide (IAA)and Tris-HCl (ACS reagent) were purchased from Sigma-Aldrich (Merck, USA). RIPAPierceTMlysis, Tris(2-carboxyethyl) phosphine (TCEP), PierceTMtrypsin, AspN protease (MS grade) and BCA Protein Assay Kit were purchased from Thermo Fisher Scientific (San Jose, CA, USA). Formic acid (HPLC grade) was bought from Macklin (Shanghai, China).

\section{Plasma and serum sample preparation}

The participants were recruited from the Peking University People's Hospital. Serum and plasma samples for research purposes were collected in pro-coagulation or in anticoagulant vacuum tubes using standard venepuncture protocols. Serum or plasma was extracted by centrifugation for $10 \mathrm{~min}$ at $3000 \mathrm{rpm}$ and subsequently stored at $-80{ }^{\circ} \mathrm{C}$ before use. 


\section{Protein extraction, reduction, alkylation and digestion}

Exosomes were lysed using RIPA buffer containing $1 \%$ protease inhibitor at $4{ }^{\circ} \mathrm{C}$ for $40 \mathrm{~min}$. After centrifugation at $14,000 \mathrm{~g}$ for $15 \mathrm{~min}$ at $4{ }^{\circ} \mathrm{C}$, the supernatant containing exosome proteins was collected. For plasma or serum samples, the high-abundance proteins were immunoisolated by HPLC with a Multi Affinity Removal Column according to the manufacturer's protocol. The proteins were precipitated with trichloroacetic acid (TCA) and then washed twice with acetone. Proteins were dissolved in $8 \mathrm{M}$ urea, then reduced with 10 $\mathrm{mM}$ tris-(2-carboxyethyl) phosphine (TCEP) for $40 \mathrm{~min}$ and alkylated with $20 \mathrm{mM}$ iodoacetamide (IAA) for $30 \mathrm{~min}$ in the dark. Protein digestion was done in two steps. First, the proteolytic enzymes AspN was added in a 1:100 ratio, and incubated at $37^{\circ} \mathrm{C}$ for $6 \mathrm{hr}$. Then, trypsin was added in a 1:50 ratio, and incubated at $37^{\circ} \mathrm{C}$ for $16-20 \mathrm{hr}$. The peptide mixture was desalted on a Monospin C18 column (GL Science,Tokyo, Japan) and dried with a SpeedVac. The dried peptide samples were stored at $-80{ }^{\circ} \mathrm{C}$ before use.

\section{LC-TIMS-MSMS analysis}

$200 \mathrm{ng}$ proteolytic digests were redissolved in $20 \mu \mathrm{L}$ of $0.1 \%$ formic acid (FA) and separated by nanoLC (nanoElute, Bruker Daltonics) with a selfpacked RP column $(250 \mathrm{~mm} \times 75 \mu \mathrm{m}$, $1.9 \mu \mathrm{m})$ at a flow rate of $300 \mathrm{~nL} / \mathrm{min}$. The mobile phase consisted of $0.1 \% \mathrm{FA}$ in water (A) and $0.1 \%$ FA in acetonitrile (B). The elution gradient used was $2 \%$ to $22 \%$ mobile phase B from 2 to $90 \mathrm{~min}, 22 \%$ to $37 \%$ mobile phase B from 90 to $100 \mathrm{~min}, 37 \%$ to $90 \%$ mobile phase B from 100 to $110 \mathrm{~min}$, and then $90 \%$ mobile phase B from $110 \mathrm{~min}$ to $120 \mathrm{~min}$. The eluates were analyzed online by a trapped ion mobility Q-TOF (timsTOF Pro, Bruker Daltonics) operating in dda-PASEF mode. Mass Range was 100 to $1700 \mathrm{~m} / \mathrm{z}$, and 1/k0 was $0.6 \mathrm{~V} \cdot \mathrm{s} / \mathrm{cm} 2$ to $1.6 \mathrm{~V} \cdot \mathrm{s} / \mathrm{cm} 2$. Capillary Voltage was $1700 \mathrm{~V}$ to stabilize the electrospray. The 
dual TIMS analyzer was operated at a locked duty cycle of $100 \%$ using equal accumulation and ramp times of $100 \mathrm{~ms}$ each. The ramp start voltage $\Delta 6$ of TIMS was set to $55 \mathrm{~V}$ to avoid possible glycan loss. Each cycle contained one MS1 scan and ten PASEF MSMS scans. Scheduling target intensity was $20000 \mathrm{cts} / \mathrm{s}$, intensity threshold was $2500 \mathrm{cts} / \mathrm{s}$. CID energy started at $20 \mathrm{eV}$ and ended at $59 \mathrm{eV}$, corresponding to the $1 / \mathrm{k} 0$ range of $0.6 \mathrm{~V} \cdot \mathrm{s} / \mathrm{cm} 2$ to 1.6 $\mathrm{V} \cdot \mathrm{s} / \mathrm{cm} 2$. Singly charged precursors were excluded by their position in the $\mathrm{m} / \mathrm{z}$-ion mobility plane.

\section{Data analysis}

All raw files were analyzed using PEAKS online software. Experiment type was set as TIMS-DDA with CID fragmentation. The data was searched in the Uniprot human protein databases (140220 entries). The maximum mass deviations of parent and fragment ions after mass recalibration were set to $20 \mathrm{ppm}$ and $0.02 \mathrm{Da}$, respectively. Trypsin and AspN were chosen as the digestion enzymes, and the maximum missed cleavage was set at 2 . Methionine oxidation was set as variable modifications, and Cysteine carbamidomethylation was set as fixed modification. Additionally, the 14 types of O-glycoforms on Ser/Thr/Tyr residues were set as variable modifications. Each valid protein hit should contain at least one unique peptide. The false discovery rates of PSM was set within a threshold value of 0.01 . The PSM was manually checked to confirm the O-glycosylation according to b- or y- characteristic fragments containing the O-glycosylated site and the glycan oxonium ions.

\section{Isolation of exosomes from cultured cells/serum}

Cultured cells: 
A549 cells were grown on 150 mm dishes (NEST) in DMEM media with 10\% FBS depleted of exosomes until they reached a confluency of 90-100\%. One batch of purification required 30 dishes. Conditioned medium was harvested from the cultured cells. All subsequent manipulations were performed at $4^{\circ} \mathrm{C}$. Cells and large debris were removed by centrifugation at $4000 \mathrm{~g}$ for $20 \mathrm{~min}$ followed by $18,000 \mathrm{~g}$ for $20 \mathrm{~min}$ in $50 \mathrm{ml}$ tubes. The supernatant was passed through a $0.2 \mu \mathrm{m}$ filter and then centrifuged at $100,000 \mathrm{~g}$ for $70 \mathrm{~min}$ at $4^{\circ} \mathrm{C}$. The crude exosome pellet was washed with $60 \mathrm{ml} \mathrm{PBS}$, followed by a second step of ultracentrifugation at $100,000 \mathrm{~g}$ for $70 \mathrm{~min}$ at $4^{\circ} \mathrm{C}$ to collect the crude exosomes in the pellet.

\section{Serum:}

Blood samples were collected from normal donors for research purposes in 6-ml procoagulation vacuum tubes using standard venepuncture protocols. Human serum was withdrawn from the tube for the purification procedures. A total of $50 \mathrm{ml}$ of cell-free serum, which was obtained by combining the serum from 50 healthy controls, was required for one batch purification. Large debris was removed by centrifugation at $1000 \mathrm{~g}$ for $10 \mathrm{~min}$ followed by $4000 \mathrm{~g}$ for $20 \mathrm{~min}$. Crude exosomes were collected by centrifugation at 100,000 g for 70 min, then washed with PBS.

Further exosome purification was performed by Iodixanol-sucrose density gradient centrifugation, using Optiprep (Sigma-Aldrich, D1556) as the density medium. The procedure can be applied to exosome purification from both cultured cells and serum. The gradient was formed by layering $1 \mathrm{~mL}$ of $40 \%(\mathrm{w} / \mathrm{v}), 1 \mathrm{~mL}$ of $20 \%(\mathrm{w} / \mathrm{v}), 1 \mathrm{~mL}$ of $10 \%(\mathrm{w} / \mathrm{v})$, and $1 \mathrm{~mL}$ of $5 \%(\mathrm{w} / \mathrm{v})$ iodixanol solutions on top of each other in a $5 \mathrm{~mL}$ open top polyallomer tube (Beckman Coulter). Crude exosomes were resuspended in $200 \mu \mathrm{L}$ PBS and overlaid on top of the gradient. The gradient was centrifuged at $100,000 \mathrm{~g}$ and $4^{\circ} \mathrm{C}$ for $18 \mathrm{~h}$. 
10 fractions of $500 \mu \mathrm{l}$ each were collected from the top and exosomes were mainly enriched in fraction 7.

\section{Negative staining}

Purified exosome pellets were resuspended in 50-100 $\mu$ l PBS, then a $5 \mu 1$ sample of each was mixed with the same volume of $2.5 \%$ glutaraldehyde (PB, $\mathrm{pH} 7.4)$, and fixed for $30 \mathrm{~min}$ at room temperature. The sample was spread onto glow discharged Formvar-coated copper mesh grids (Electron Microscopy Sciences, Hatfield) for about 5 min, then washed with water. The sample was then stained with uranyl acetate for 2 min. Excess staining solution was blotted off with filter paper and copper mesh grids were washed with water. Post drying, grids were imaged at $10-100 \mathrm{kV}$ using a transmission electron microscope $\mathrm{H}-7650$.

\section{Statistics:}

The quantification of the PSM of O-glycosylation in Figure 4 was done as follows: three technical replicates were done for each sample. To avoid randomness of O-glycosylation detection, the PSM of a certain O-glycopeptide and the total PSM of the corresponding peptides from the 3 technical replicates were added together. The O-glycosylation state of each O-glycopeptide was calculated by normalizing the O-glycopeptide PSM to the total PSM of the corresponding peptides. Statistical analysis was performed in GraphPad Prism. Error bars in the figures represent the mean \pm s.e.m. Comparisons were performed by twotailed, unpaired Student's t-tests. $\mathrm{P}<0.05$ was considered statistically significant. We developed software for the calculations mentioned above for all the identified Oglycopeptides. 


\section{Acknowledgement:}

We thank Dr. Ning Chen of Bruker Daltonics, Inc. Billerica, MA, for her valuable contributions and ongoing support in developing the TIMS-TOF mass spectrometry applications in our laboratory. We thank Dr. Dawei Liu of Crystlab Tech Corp., Beijing, for his constant support with data analysis. We thank Dr. Ying Li of the Cell Facility in the Center of Biomedical Analysis of Tsinghua University for help with electron microscopy. The research was supported by grants from the Fundamental Research Funds for the Central Universities BMU2017YJ003, BMU2018XTZ002 to Catherine CL Wong, the PKU-Baidu Fund 2019BD007 to Catherine CL Wong, the Ministry of Science and Technology of the People's Republic of China 2018YFA0507102 to Yang Chen and the National Natural Science Foundation of China 91754108 and 31671395 to Yang Chen. Catherine CL Wong thanks the Research Funds from Health@InnoHK Program launched by Innovation Technology Commission of the Hong Kong Special Administrative Region.

\section{Author contributions:}

C.CL.W and Y.C designed the experiments. FL.H, H.Z and XJ.H collected clinical samples. X.S, JH.C, WM.T and JT.G conducted the experiments. X.S, WM.T, JH.C, SX.G, FL.H, H.Z, XJ.H, C.CL.W and Y.C analyzed the data. C.CL.W and Y.C wrote the manuscript.

\section{Conflict of interests:}

The authors declare no conflict of interests. 


\section{Figure legends:}

\section{Figure 1 - Enrichment-Free Identification of Native Definitive (EnFIND) O- glycoproteome}

A. Workflow of EnFIND.

B. Summary of the number of identified proteins, O-glycoproteins, O-glycopeptides and Oglycosites. 5\% false discovery rate (FDR) at the protein level was used as the cut-off.

C. Overlap of the O-glycoproteins identified in this study with previously published Oglycoproteins.

D. The relative proportion of serine/tyrosine/threonine $(\mathrm{S} / \mathrm{Y} / \mathrm{T}) \mathrm{O}$-glycosites identified in this study.

E. Histograms of the O-glycoproteome, O-glycoproteome peptides, and O-glycopeptides. Yaxis is the percentage of protein or peptides number to total protein or peptides number; $\mathrm{X}$ axis is the intensity. The proteins or peptides with intensity lower than $2 \mathrm{e}+02$ are indicated by an asterisk.

F. Systematic illustration of O-glycoforms selected in this study. O-glycoforms reported in the literature are indicated by an asterisk.

G. The distribution of 14 types of O-glycoform in individual samples.

\section{Figure 2 - Features of the EnFIND method.}

A. Ion mobility separation of the O-glycopeptide DSKPDTtAPPSSPK $\left([\mathrm{M}+3 \mathrm{M}]^{3+}, 815.895\right.$ $\mathrm{m} / \mathrm{z})$ and the unmodified peptide DSKPDTTAPPSSPK $\left([\mathrm{M}+3 \mathrm{M}]^{3+}, 714.355 \mathrm{~m} / \mathrm{z}\right)$. The mass of GlcNAc (blue square) or GalNAc (yellow square) is 203.08). Upper panel: Extracted ion chromatogram (EIC) and extracted ion mobilogram (EIM). (blue: DSKPDTTAPPSSPK; 
orange: DSKPDTtAPPSSPK). Lower panel: MS/MS spectra of DSKPDTtAPPSSPK (Opeptide) and DSKPDTTAPPSSPK (peptide). The characteristic peaks are indicated by an asterisk.

B. Ion mobility separation of two isomeric O-glycopeptides, NDItAVTKEGK and NDITAVtKEGK $\left([\mathrm{M}+3 \mathrm{M}]^{3+}, 661.343 \mathrm{~m} / \mathrm{z}\right)$. The mass of Fucose is 146.06 (red triangle). Upper panel: EIC and EIM of NDItAVTKEGK (green) and NDITAVtKEGK (red); Lower panel: MS/MS spectra of NDItAVTKEGK (O-peptide1) and NDITAVtKEGK (O-peptide 2). The characteristic peaks are indicated by an asterisk.

C. Determination of different glycan structures of isomeric O-linked trisaccharide (mass = 511.19) glycopeptides.

Panel a: MS/MS spectrum of DYYCLsG $\left([\mathrm{M}+2 \mathrm{M}]^{2+}, 694.766 \mathrm{~m} / \mathrm{z}\right)(\mathrm{O}-\mathrm{peptide})$. The characteristic peaks are indicated by an asterisk.

Panel b: EIC of DYYCLsG $\left([\mathrm{M}+2 \mathrm{M}]^{2+}, 694.766 \mathrm{~m} / \mathrm{z}\right)$

Panel c: EIM of the total separation of DYYCLsG. 1 (Mobility, 1/K0 = 0.953), 2 (Mobility, $1 / \mathrm{K} 0=1.006$ ), and $\mathbf{3}$ (Mobility, 1/K0 = 1.220) indicate different glycan isomers at the site.

Panel d: MS/MS spectrum of 1 (CID fragment ions of $349.16 \mathrm{~m} / \mathrm{z}$ and $146.13 \mathrm{~m} / \mathrm{z}$, corresponding to the sequentially loss of a Gal/Glc/Man and GlcNAc/GalNAc residues).

Panel e: MS/MS spectrum of 2 (CID fragment ions of $365.13 \mathrm{~m} / \mathrm{z}$ and $308.11 \mathrm{~m} / \mathrm{z}$, corresponding to the loss of Fucose and GlcNAc/GalNAc residues).

Panel f: MS/MS spectrum of 3 (CID fragment ions of $349.15 \mathrm{~m} / \mathrm{z}$ and $203.15 \mathrm{~m} / \mathrm{z}$, corresponding to the sequentially loss of a Gal/Glc/Man and Fucose residues).

D. Proposed cleavage mechanism maps for the generation of various linkage-diagnostic fragments from the trisaccharide (mass $=511.19)$ glycopeptides of $\mathbf{1}(1 / \mathrm{K} 0=0.953), 2$ 
$(1 / \mathrm{K} 0=1.006)$ and $\mathbf{3}(1 / \mathrm{K} 0=1.220)$. The components of the trisaccharide are fucose (red color, mass $=146.07)$, GlcNAc/GalNAc (blue color, mass = 203.15) and Gal/Glc/Man (yellow color, mass $=162.05)$

\section{Figure 3 - Identification of O-glycosylation on antibodies}

A. A schematic overview of identified O-glycosylated antibody from serum and serum exosomes. O-glycosylation sites with multiple glycoforms were identified in the variable domain, constant domain and hinge region.

B. Summary of total O-glycosylations of antibody in variable and constant regions.

C. Summary of total number of O-glycosylation sites in different categories of antibody in human serum samples.

D. Summary of isomeric O-glycopeptides in CDRs.

E, F. Average numbers of antibody O-glycosites (E) or O-glycosites and O-glycoforms (F) identified in two healthy controls. Each sample was repeatedly analyzed 13 times.

G, H, I. Comparison of identified antibodies (G), antibody O-glycosites $(\mathrm{H})$ and antibody Oglycosites and O-glycoforms (I) in two healthy controls. The data were the union of 13 analyses.

\section{Figure 4 - Antibody O-glycosylation is correlated with autoimmune diseases}

A, B. Summary of statistically different O-glycosylations in patients with SLE (A) and mild or critical IgA Nephropathy (B) compared to healthy controls by two-tailed, unpaired Student's t-tests. $* \mathrm{P}<0.05, * * \mathrm{P}<0.01, * * * \mathrm{P}<0.001$.

C, D. Scatter plot showing O-glycosites which distinguish RA and SS (C) or mild and critical cases of IgA nephropathy (D). The PSM of O-glycosylated peptide of indicated sites from 3 
technical repeats were added and then normalized to total PSM of the indicated peptides added from 3 technical repeats. Individual normalized values for the indicated disease cases and healthy controls are shown. Mean values \pm S.E.M, Comparisons were performed by twotailed, unpaired Student's t-tests. $* \mathrm{P}<0.05, * *, \mathrm{P}<0.01$.

\section{Expanded view Figures legends:}

Fig EV1 Exosomes purified from cultured A549 cells or serum.

(A) Purified exosomes were analyzed by western blotting using antibodies against the exosome specific markers Alix, Tsg101, CD63 and Flotilin.

(B) Representative TEM images of negatively stained samples of exosomes. Scale bar, 500 nm.

Fig EV2 Venn diagram of O-glycoproteins identified in A549 cell lysate and exosomes derived from A549 (left), and serum and exosomes purified from serum (right).

Fig EV3 GO analysis of identified O-glycosylated proteins. For GO analysis, the glycoproteins identified in A549 and A549-derived exosomes were combined (A549 \& exo) and the glycoproteins identified in serum and exosomes from serum were combined (Serum \& exo).

Fig EV4 Analysis of amino acid sequences in CDR1 and CDR2 from the IMGT database. The size of the letter represents the frequency of the amino acid.

Fig EV5 MS2 spectrum of all identified isomeric glycoforms on antibody CDRs.

Fig EV6 Scatter plot graph of O-glycosites in patients with SLE and mild and critical IgA nephropathy compared to healthy controls. The PSM of O-glycosylated peptide of indicated 
sites from 3 technical repeats were added and then normalized to total PSM of the indicated peptides added from 3 technical repeats. Individual normalized values for the indicated disease cases and healthy controls are shown. Mean values \pm S.E.M, Comparisons were performed by two-tailed, unpaired Student's t-tests. * $\mathrm{P}<0.05$, **, $\mathrm{P}<0.01$, ***, $\mathrm{P}<0.001$.

\section{Expanded view table legends:}

\section{Table EV1}

Numbers of O-glycoforms in the indicated regions of antibodies in human serum.

\section{Table EV2}

Numbers of O-glycosites in the indicated regions of different antibody isotypes

\section{Table EV3}

Characteristics of the disease and control cohorts.

\section{Table EV4}

Raw data of the O-glycosylations including total PSM, total O-glycopeptide PSM of the Oglycopeptides mentioned in the study. 


\section{References:}

Arnold JN, Wormald MR, Sim RB, Rudd PM, Dwek RA (2007) The impact of glycosylation on the biological function and structure of human immunoglobulins. Annu Rev Immuno/25: 21-50

Chai W, Zhang Y, Mauri L, Ciampa MG, Mulloy B, Sonnino S, Feizi T (2018) Assignment by Negative-Ion Electrospray Tandem Mass Spectrometry of the Tetrasaccharide Backbones of Monosialylated Glycans Released from Bovine Brain Gangliosides. J Am Soc Mass Spectrom 29: 1308-1318

Darula Z, Sarnyai F, Medzihradszky KF (2016) O-glycosylation sites identified from mucin core-1 type glycopeptides from human serum. Glycoconj J 33: 435-445

de Haan N, Falck D, Wuhrer M (2020) Monitoring of immunoglobulin N- and Oglycosylation in health and disease. Glycobiology 30: 226-240

Halim A, Nilsson J, Ruetschi U, Hesse C, Larson G (2012) Human urinary glycoproteomics; attachment site specific analysis of $\mathrm{N}$ - and O-linked glycosylations by CID and ECD. Mol Cell Proteomics 11: M111 013649

Hoffmann M, Marx K, Reichl U, Wuhrer M, Rapp E (2016) Site-specific OGlycosylation Analysis of Human Blood Plasma Proteins. Mol Cell Proteomics 15: 624-641

Joshi HJ, Narimatsu Y, Schjoldager KT, Tytgat HLP, Aebi M, Clausen H, Halim A (2018) SnapShot: O-Glycosylation Pathways across Kingdoms. Cell 172: 632-632 e632 
King SL, Joshi HJ, Schjoldager KT, Halim A, Madsen TD, Dziegiel MH, Woetmann A, Vakhrushev SY, Wandall HH (2017) Characterizing the O-glycosylation landscape of human plasma, platelets, and endothelial cells. Blood Adv 1: 429-442

Levery SB, Steentoft C, Halim A, Narimatsu Y, Clausen H, Vakhrushev SY (2015) Advances in mass spectrometry driven O-glycoproteomics. Biochim Biophys Acta 1850: $33-42$

Mao J, You X, Qin H, Wang C, Wang L, Ye M (2019) A New Searching Strategy for the Identification of O-Linked Glycopeptides. Anal Chem 91: 3852-3859

Marino K, Bones J, Kattla JJ, Rudd PM (2010) A systematic approach to protein glycosylation analysis: a path through the maze. Nature chemical biology 6: 713-723

Meier F, Beck S, GrassI N, Lubeck M, Park MA, Raether O, Mann M (2015) Parallel Accumulation-Serial Fragmentation (PASEF): Multiplying Sequencing Speed and Sensitivity by Synchronized Scans in a Trapped Ion Mobility Device. J Proteome Res 14: 5378-5387

Nilsson J, Ruetschi U, Halim A, Hesse C, Carlsohn E, Brinkmalm G, Larson G (2009) Enrichment of glycopeptides for glycan structure and attachment site identification. Nat Methods 6: 809-811

Reily C, Stewart TJ, Renfrow MB, Novak J (2019) Glycosylation in health and disease. Nature reviews Nephrology 15: 346-366

Schjoldager KT, Vakhrushev SY, Kong Y, Steentoft C, Nudelman AS, Pedersen NB, Wandall HH, Mandel U, Bennett EP, Levery SB, Clausen H (2012) Probing isoformspecific functions of polypeptide GalNAc-transferases using zinc finger nuclease glycoengineered SimpleCells. Proc Natl Acad Sci U S A 109: 9893-9898 
Sheikh MO, Halmo SM, Wells L (2017) Recent advancements in understanding mammalian O-mannosylation. Glycobiology 27: 806-819

Tang Y, Wei J, Costello CE, Lin C (2018) Characterization of Isomeric Glycans by Reversed Phase Liquid Chromatography-Electronic Excitation Dissociation Tandem Mass Spectrometry. J Am Soc Mass Spectrom 29: 1295-1307

Vakhrushev SY, Steentoft C, Vester-Christensen MB, Bennett EP, Clausen H, Levery SB (2013) Enhanced mass spectrometric mapping of the human GalNActype O-glycoproteome with SimpleCells. Mol Cell Proteomics 12: 932-944

Vale AM, Nobrega A, Schroeder HW, Jr. (2015) The role of evolutionarily conserved germ-line $\mathrm{DH}$ sequence in B-1 cell development and natural antibody production. Ann N Y Acad Sci 1362: 48-56

Yang W, Ao M, Hu Y, Li QK, Zhang H (2018) Mapping the O-glycoproteome using site-specific extraction of O-linked glycopeptides (EXoO). Mol Syst Bio/ 14: e8486

Yang Z, Halim A, Narimatsu Y, Jitendra Joshi H, Steentoft C, Schjoldager KT, Alder Schulz M, Sealover NR, Kayser KJ, Paul Bennett E, Levery SB, Vakhrushev SY, Clausen H (2014) The GaINAc-type O-Glycoproteome of CHO cells characterized by the SimpleCell strategy. Mol Cell Proteomics 13: 3224-3235

Ye Z, Mao Y, Clausen H, Vakhrushev SY (2019) Glyco-DIA: a method for quantitative O-glycoproteomics with in silico-boosted glycopeptide libraries. Nature methods 16: 902-910 
bioRxiv preprint doi: https://doi.org/10.1101/2020.07.15.204511; this version posted July 15, 2020. The copyright holder for this preprint (which was not certified by peer review) is the author/funder. All rights reserved. No reuse allowed without permission.

Yu X, Huang Y, Lin C, Costello CE (2012) Energy-dependent electron activated dissociation of metal-adducted permethylated oligosaccharides. Anal Chem 84:

7487-7494 
Fig 1 Enrichment-Free Identification of Native Definitive (EnFIND) Oglycoproteome

A

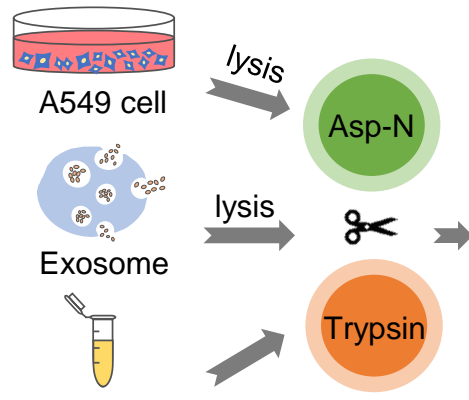

Serum

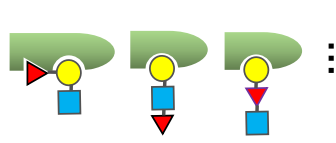

Ion position

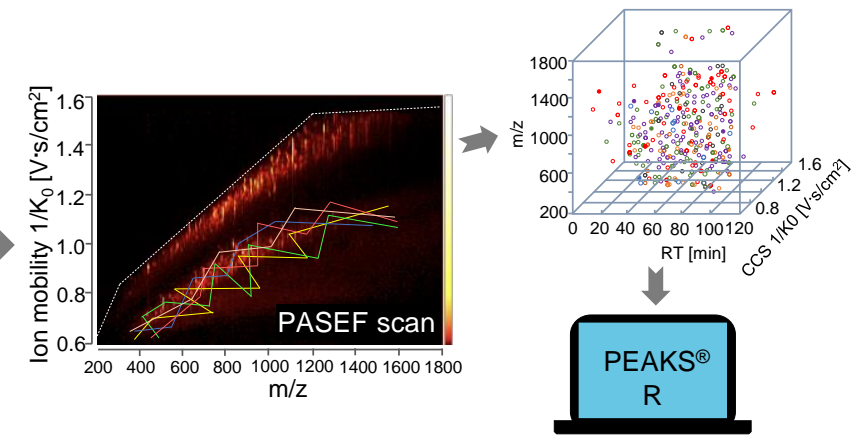

B

\section{Samples}

A549 cell

A549 exosome

Serum

Serum exosome
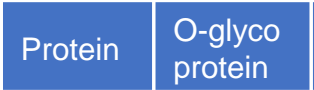

\begin{tabular}{|c|c|}
\hline $\begin{array}{c}\text { O-glyco } \\
\text { peptide }\end{array}$ & \multicolumn{1}{|l|}{$\begin{array}{l}\text { O-glyco } \\
\text { site }\end{array}$} \\
\hline 2896 & 3542 \\
\hline 366 & 456 \\
\hline 1427 & 2059 \\
\hline 1172 & 1553 \\
\hline
\end{tabular}

C This study (2462)

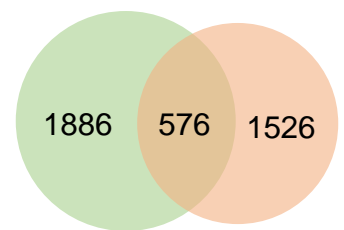

$D$

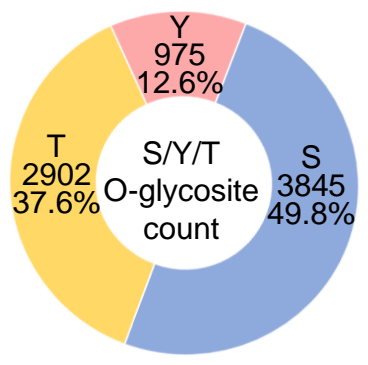

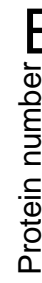
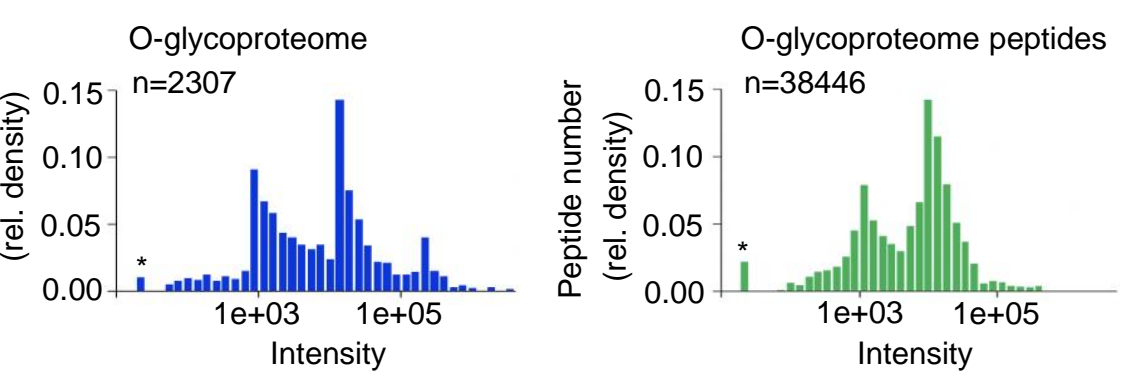

F

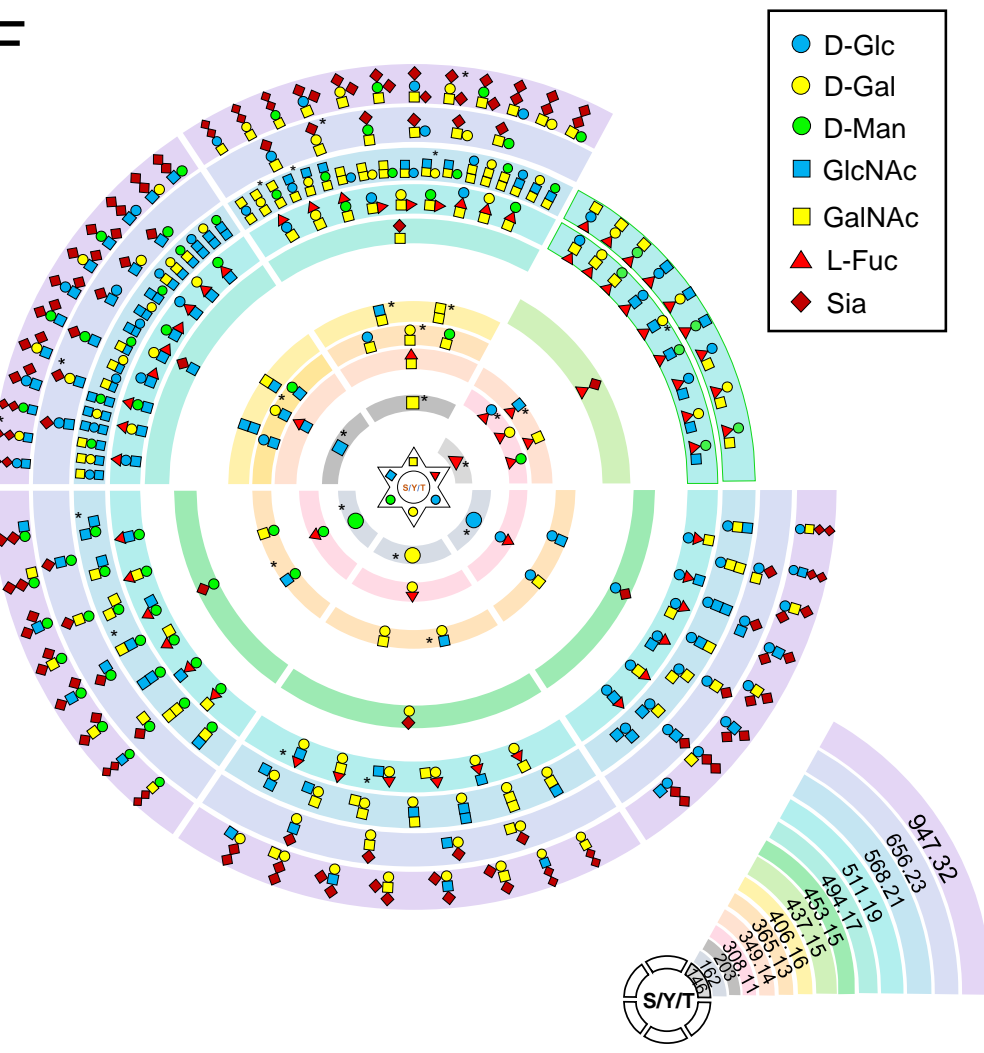

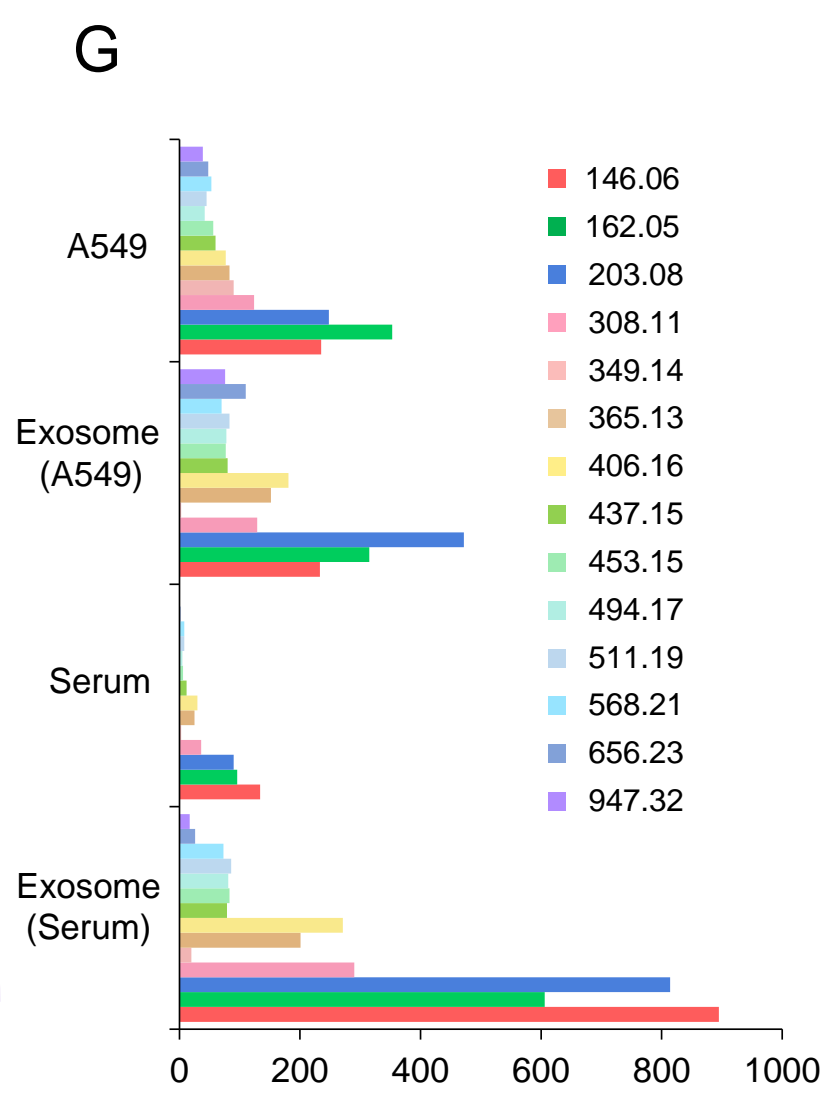

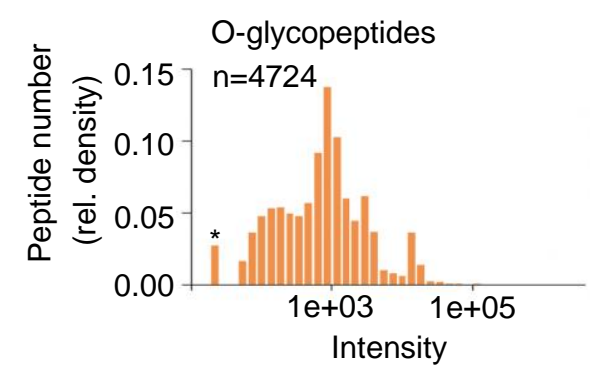

* proteins/peptide below LOQ 


\section{Fig 2 Features of the EnFIND method}

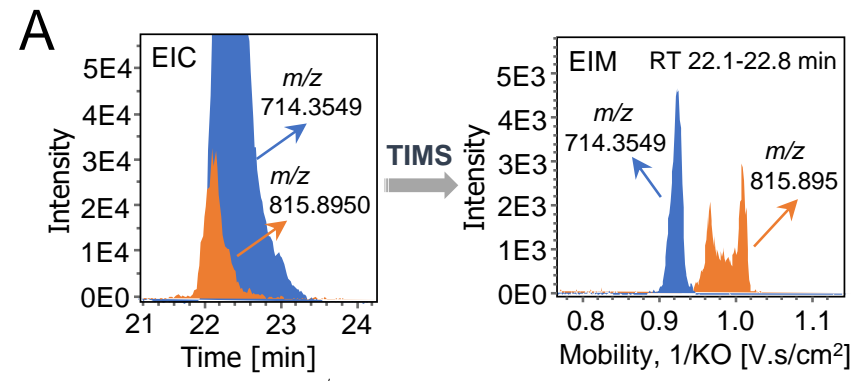

O-peptide: $\mathbf{D} \sqrt{\mathbf{S}} \sqrt{\mathbf{K}} \sqrt{\mathbf{P}} \sqrt{\mathbf{D}} \sqrt{\mathbf{T}} \sqrt{\mathbf{t}} \sqrt{\mathbf{A}} \sqrt{\mathbf{P}} \sqrt{\mathbf{P}} \sqrt{\mathrm{S}} \sqrt{\mathrm{S}} \sqrt{\mathbf{P K}}$
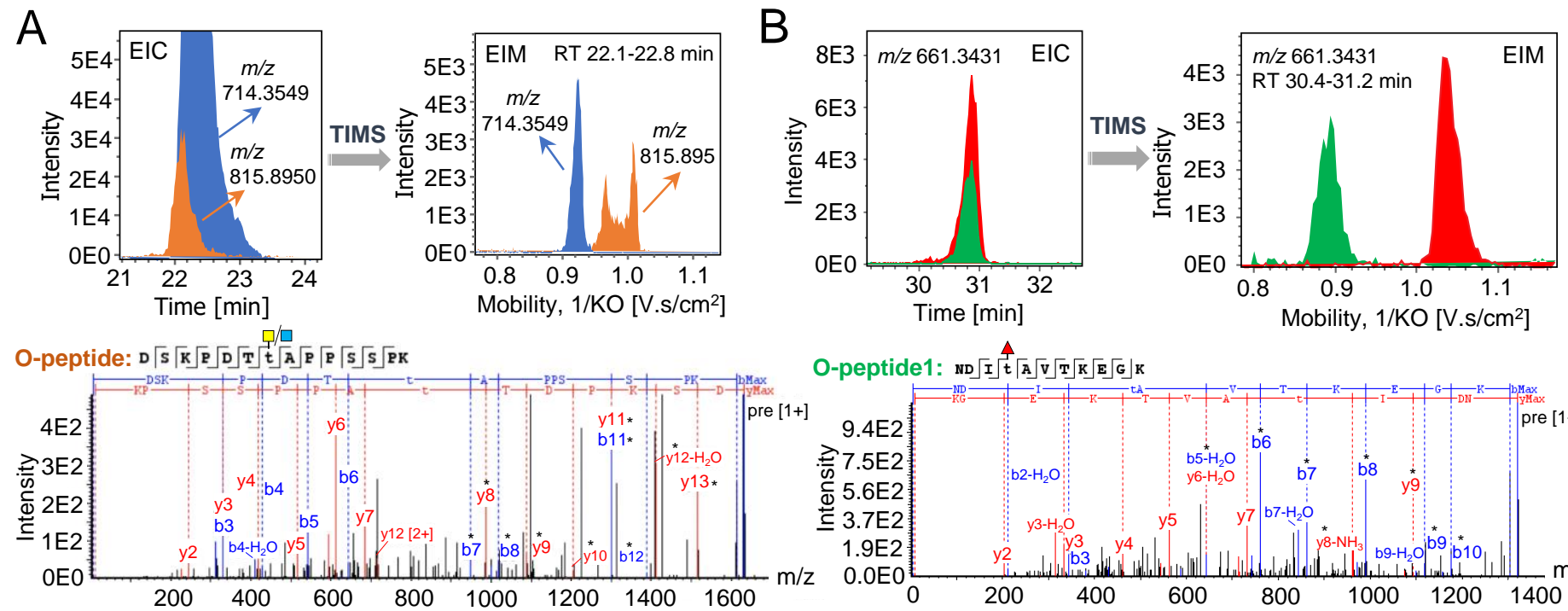

O-peptide1: $\mathbf{N D} \sqrt{\mathbf{I}} \sqrt{\mathbf{t}} \sqrt{\mathbf{A}} \sqrt{\mathbf{V}} \sqrt{\mathbf{T}} \sqrt{\mathbf{K}} \sqrt{\mathbf{E}} \sqrt{\mathbf{G}}\rfloor \mathbf{K}$

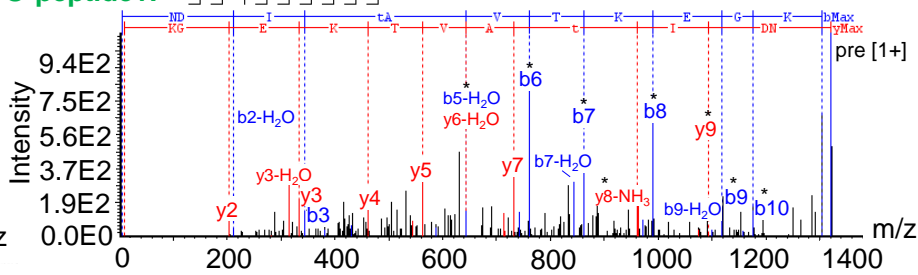

Peptide: $\quad \mathbf{D}\lceil\mathbf{S} \sqrt{\mathbf{K}} \sqrt{\mathbf{P}} \sqrt{\mathbf{D}} \sqrt{\mathbf{T}} \sqrt{\mathbf{T}} \sqrt{\mathbf{A}} \sqrt{\mathbf{P}} \sqrt{\mathbf{P}} \sqrt{\mathrm{S}} \sqrt{\mathbf{S}} \sqrt{\mathbf{P}}\rfloor \mathbf{K}$

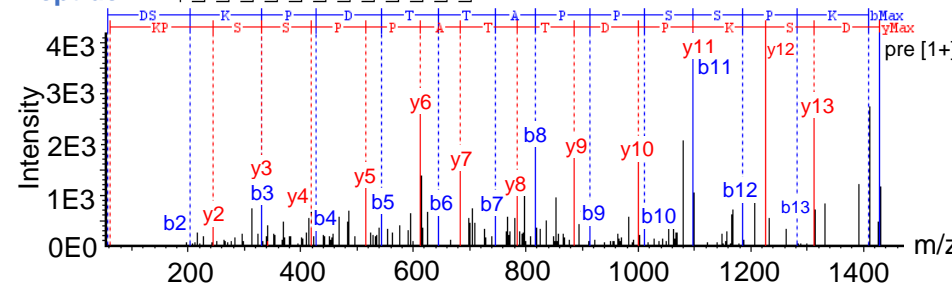

O-peptide2: $\mathbf{x} \sqrt{\mathbf{D}}\rfloor \mathbf{I} / \mathbf{T} \sqrt{\mathbf{A}} \sqrt{\mathbf{v}} \sqrt{\mathbf{t}} \sqrt{\mathbf{K}} \sqrt{\mathbf{E}} \sqrt{\mathbf{G}} \mid \mathbf{K}$

O

Panel a

O-peptide: $\mathrm{DY} \sqrt{\mathrm{Y}}[\mathrm{c}[\overline{\mathrm{I}}[\mathrm{S}] \mathrm{G}$

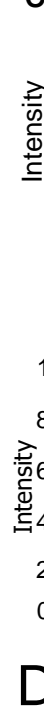

Panel d
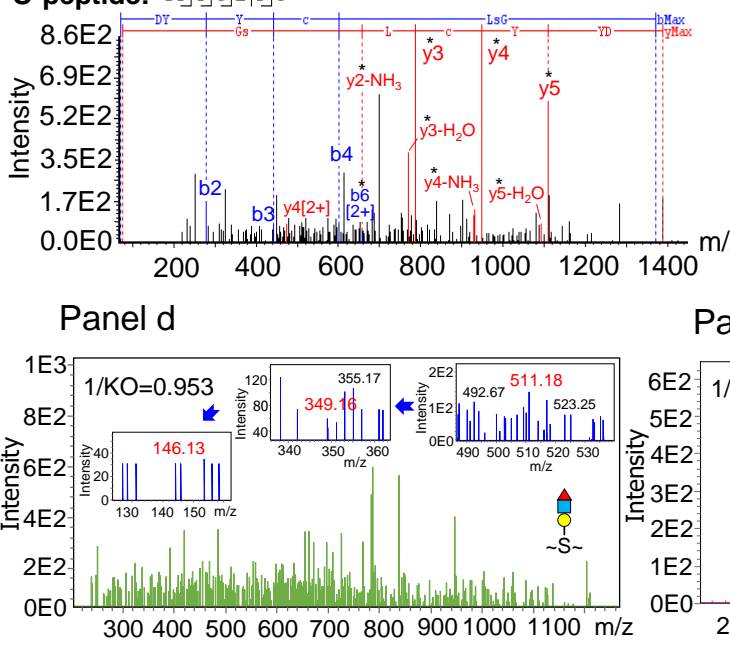

Panel e

\section{Panel b}

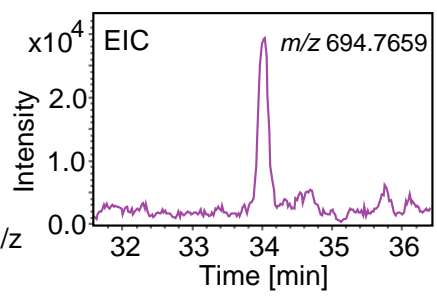

\section{$1 / \mathrm{KO}=1.006$}

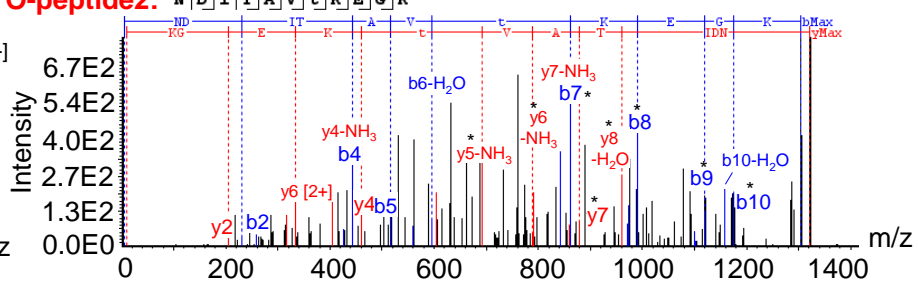

Panel c

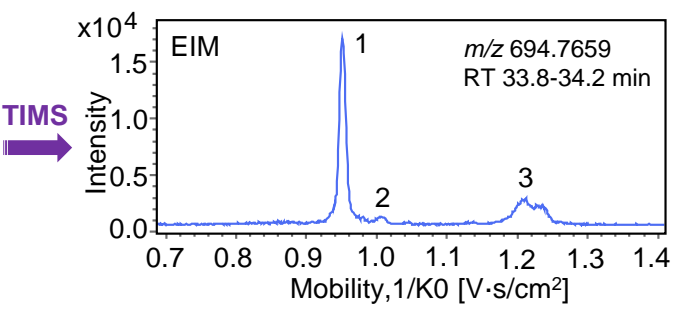

Panel $f$

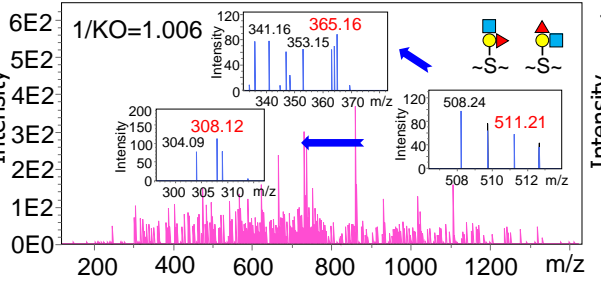
$1 / \mathrm{KO}=0.953$

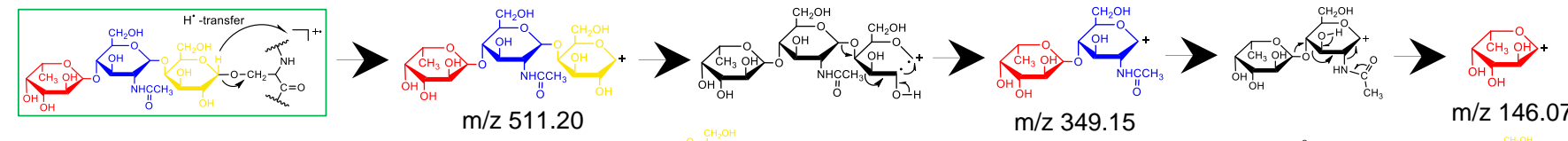

ine: 1006 (6)

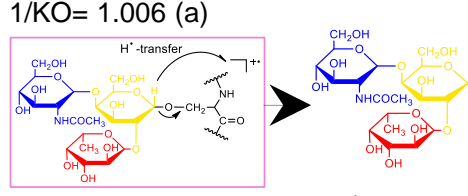

$1 / \mathrm{KO}=1.220$

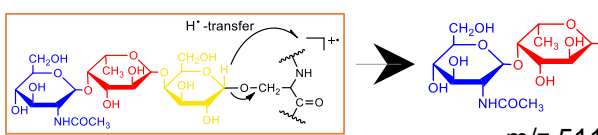

$\mathrm{m} / \mathrm{z} 511.20$

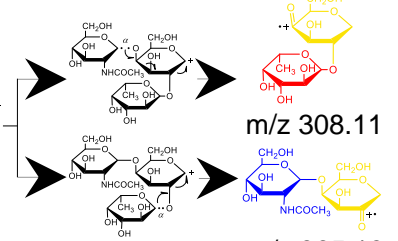

$\mathrm{m} / \mathrm{z} 365.13$
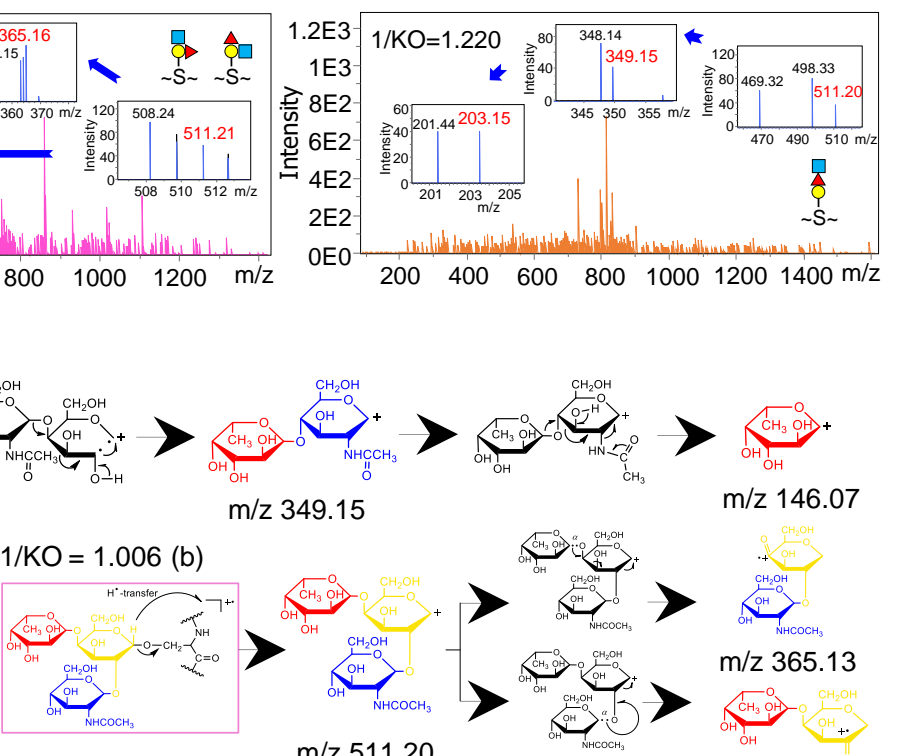

$\mathrm{m} / \mathrm{z} 308.11$

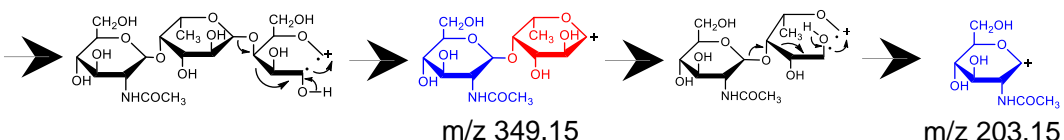


Fig 3 Identification of O-glycosylation on antibodies

A

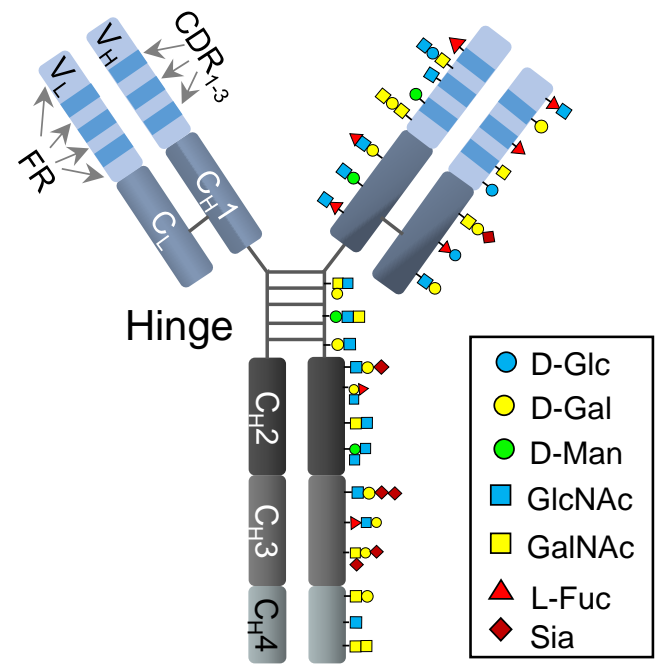

C

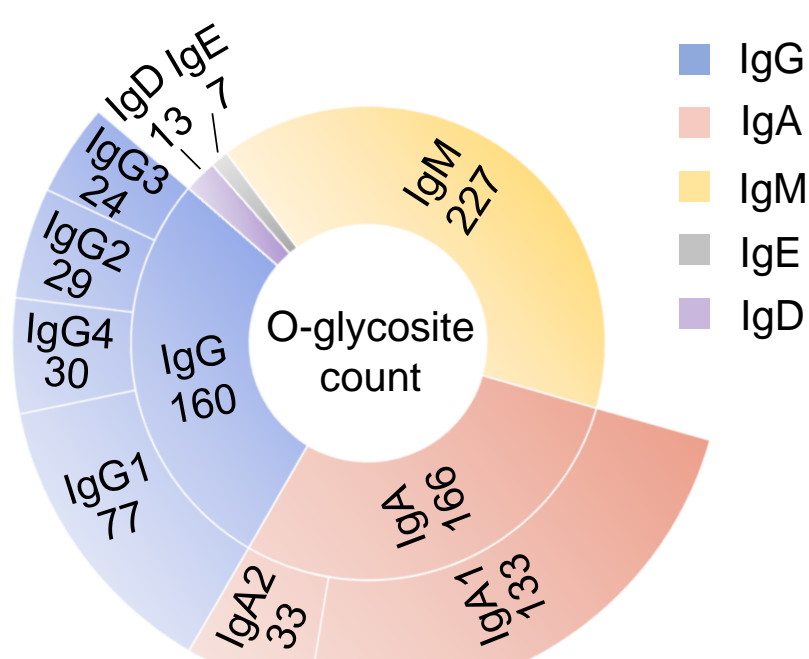

B
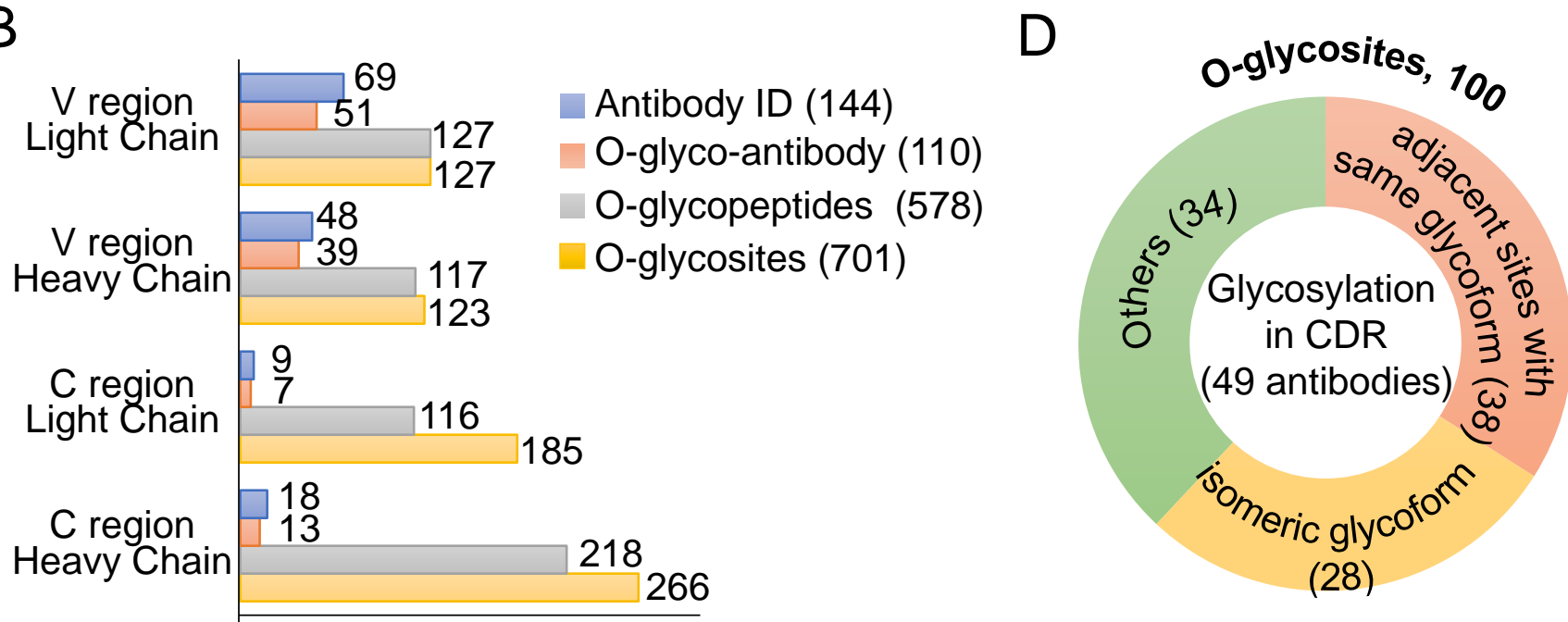

E

Number
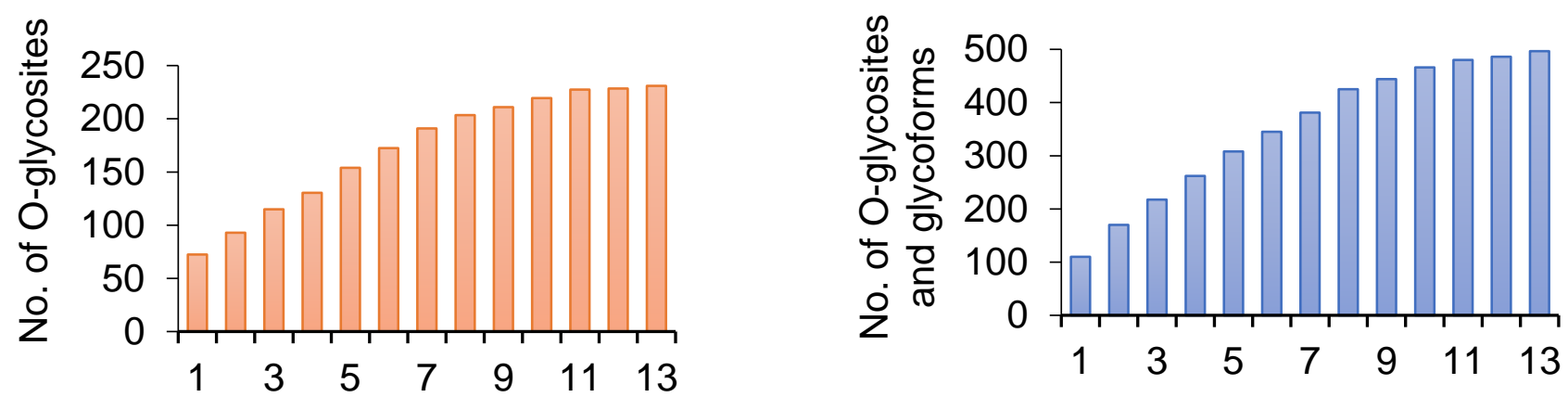

G

$\mathrm{H}$

Total antibody

Sample 1 Sample 2

105

115
Antibody O-glycosites

Sample 1 Sample 2 185

277

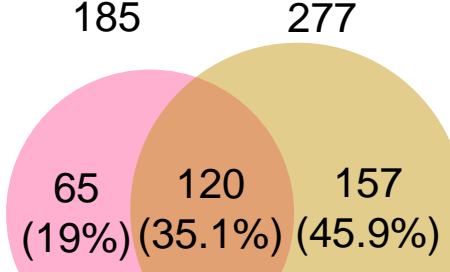

Antibody O-glycosites and glycoforms

Sample 1 Sample 2 420

573

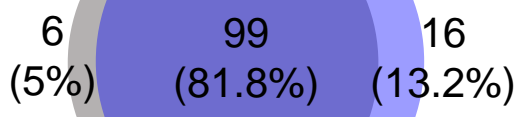


Fig 4 Antibody O-glycosylation is correlated with autoimmune diseases
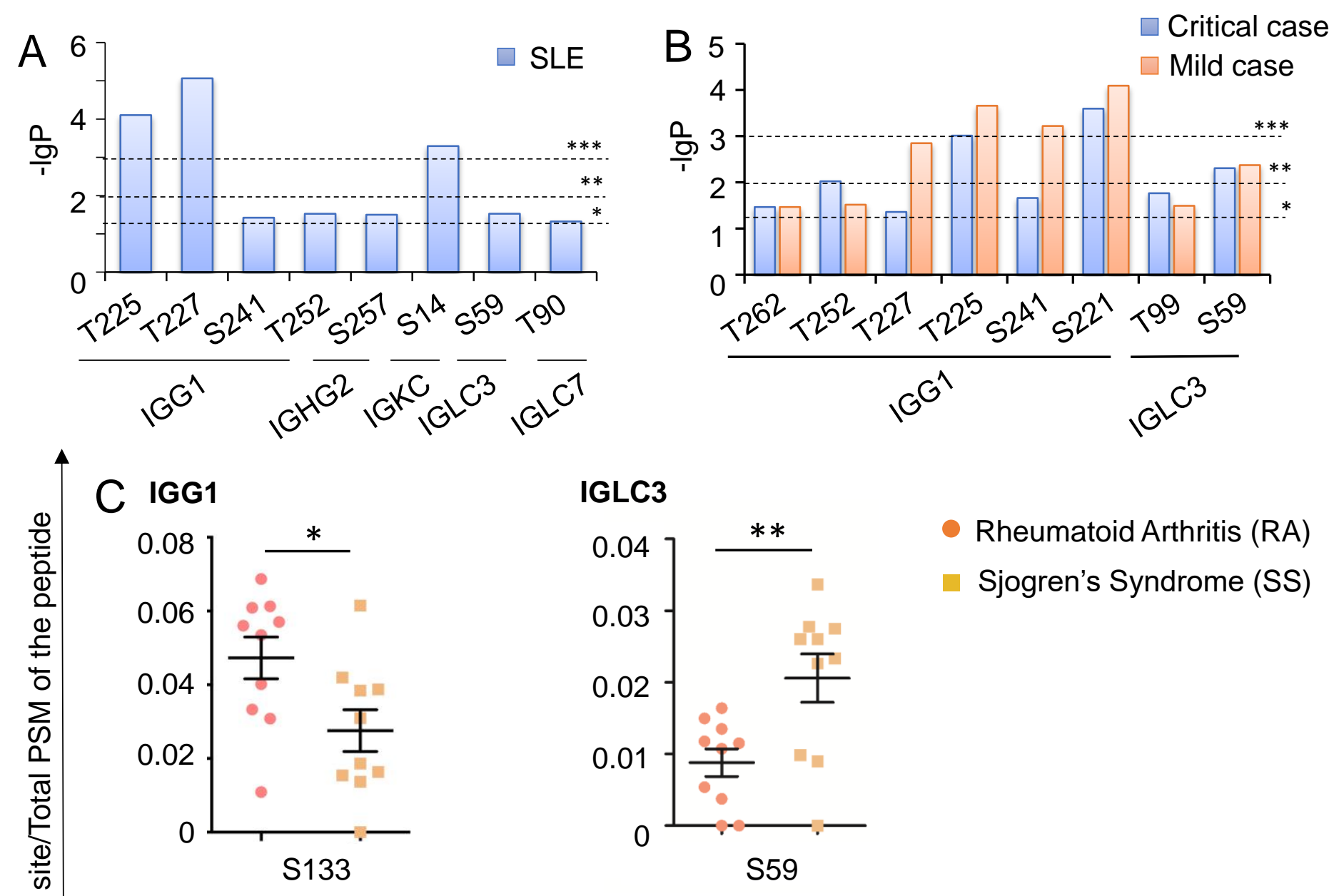

IGLC3

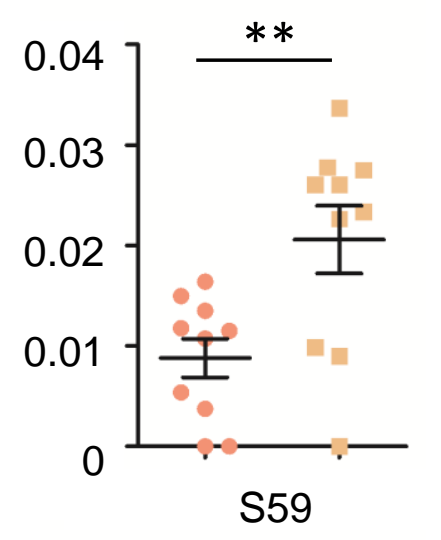

IGKC

IGKC
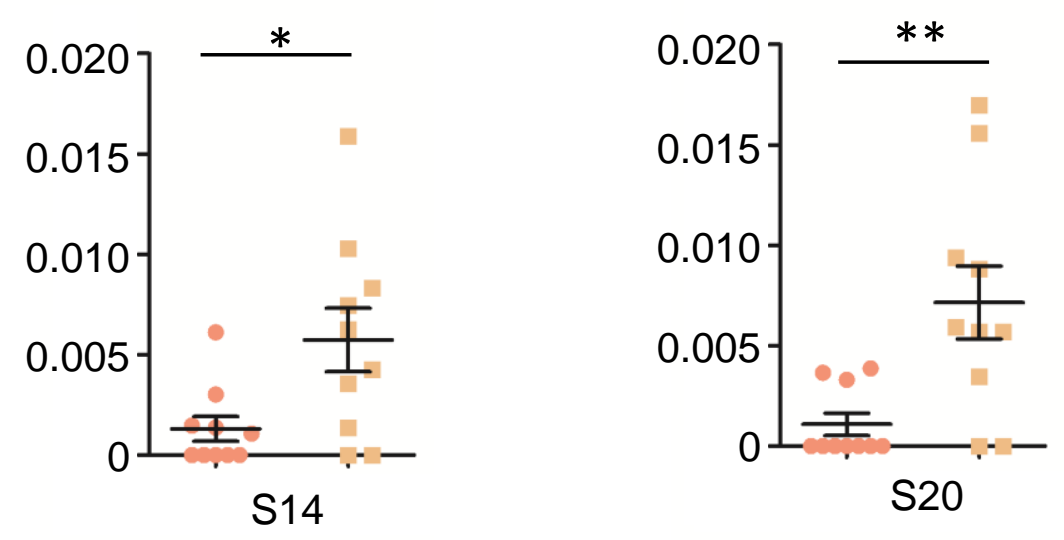

D

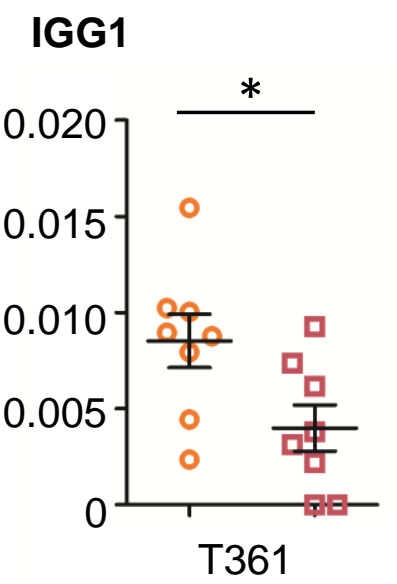

IGLC3

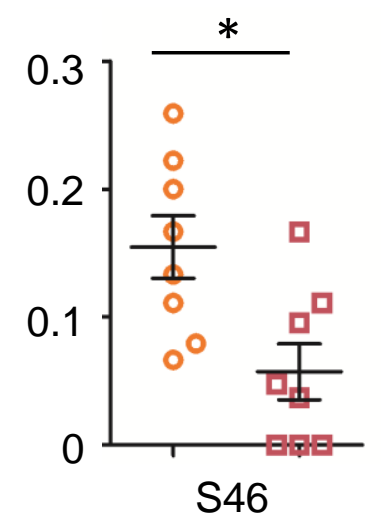

Rheumatoid Arthritis (RA) Sjogren's Syndrome (SS)
IGLC7

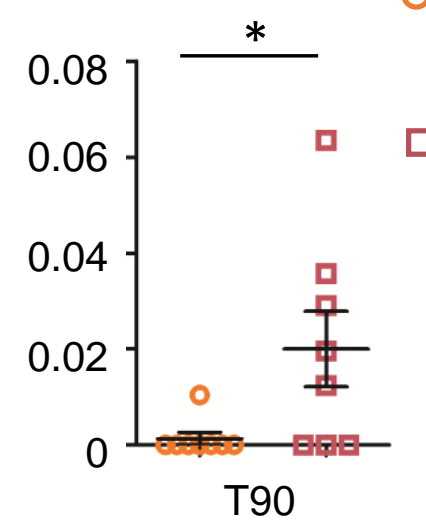

$\lg$ A nephropathy -mild case

$\lg$ A nephropathy -critical case 
Fig EV1 Exosomes purified from A549 or serum

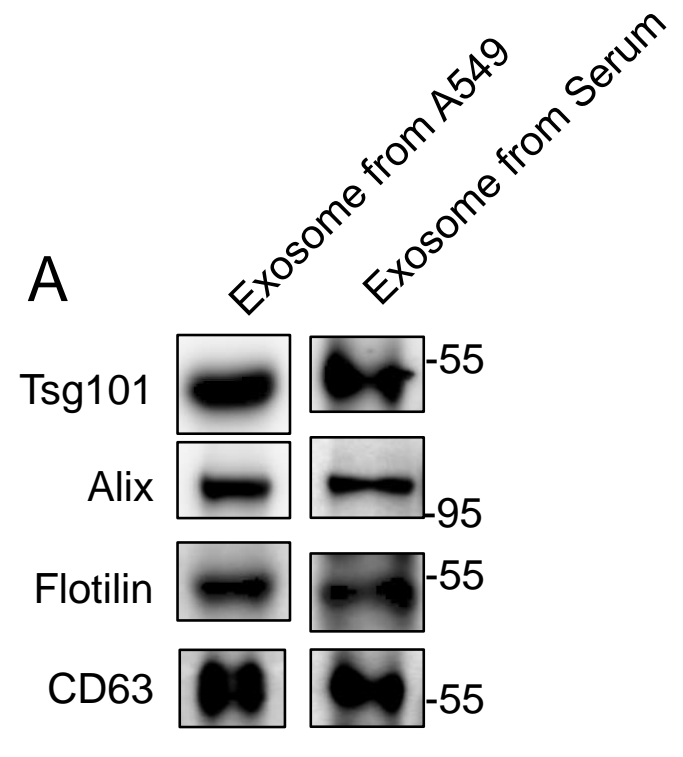

B

Exosome from A549 Exosome from Serum

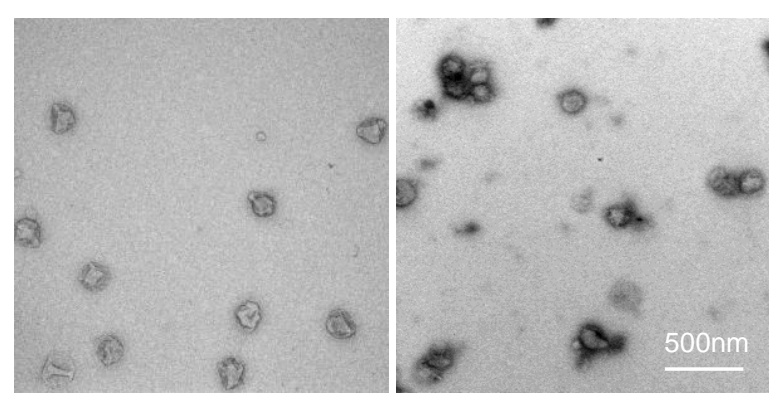


Fig EV2 Venn diagram of O-glycoproteins identified in A549 cell lysate, exosome derived from A549, serum and exosome purified from serum.

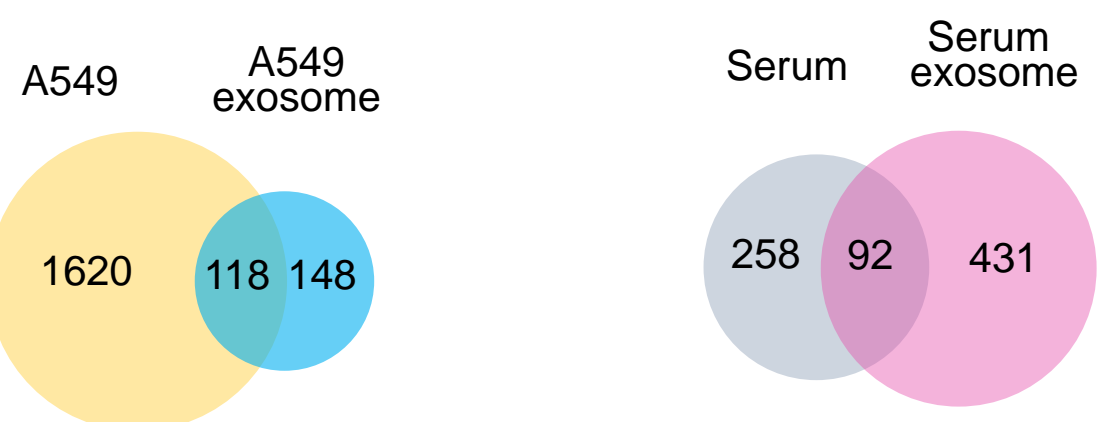




\section{Fig EV3 GO analysis of identified O-glycosylated proteins}
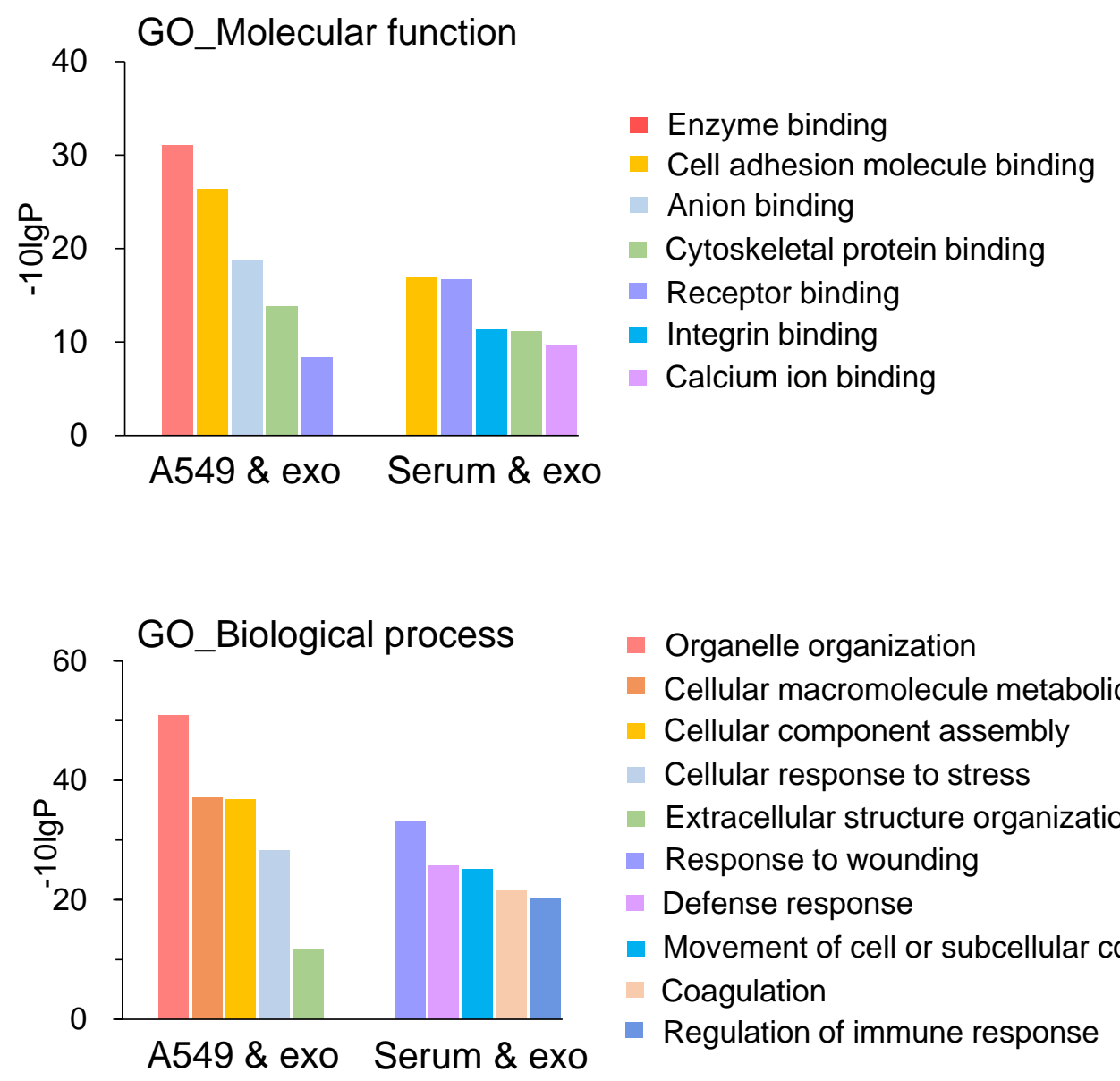

- Organelle organization

- Cellular macromolecule metabolic process

- Cellular component assembly

- Cellular response to stress

- Extracellular structure organization

- Response to wounding

- Defense response

- Movement of cell or subcellular component

- Coagulation

- Regulation of immune response

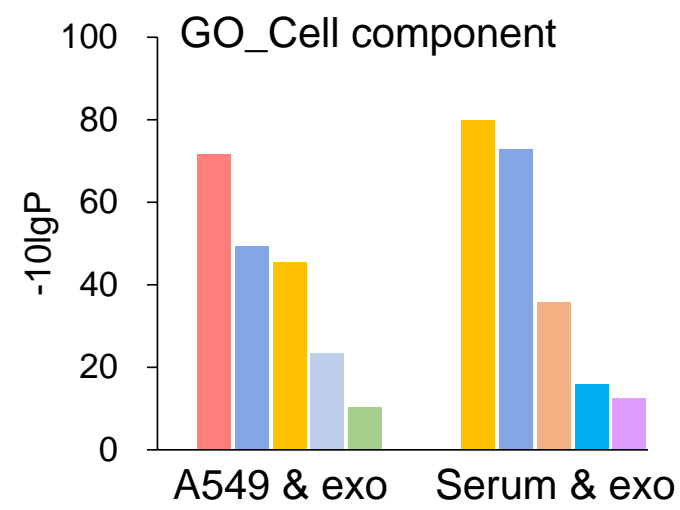
Cytoplasm
Vesicle
Extracellular exosome
Adherens junction
Endoplasmic reticulum membrane
- Blood microparticle
Secretory granule
- Proteinaceous extracellular matrix 
Fig EV4 Analysis of amino acid sequences in CDR1 and CDR2 from the IMGT database. The size of the letter represents the frequency of the amino acid.

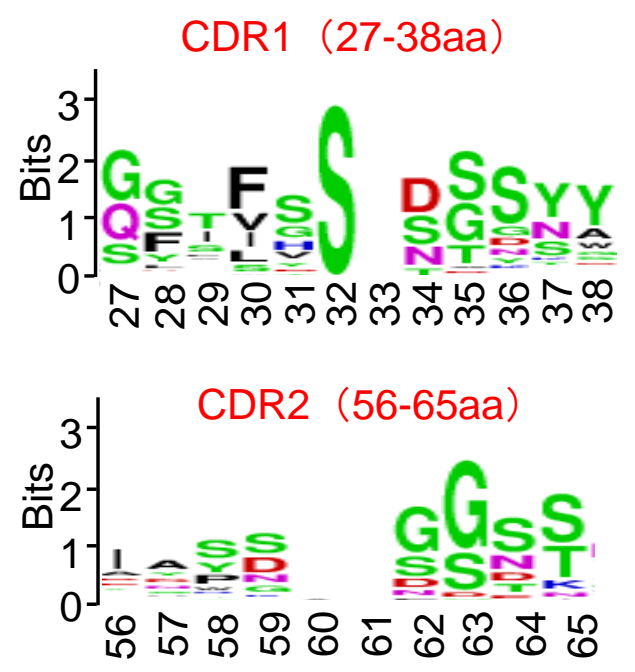


Fig EV5 MS2 spectrum of all identified isomeric glycoforms on antibody CDRs.

See word file named "Fig EV5" 
Fig EV6 Scatter plot graph of O-glycosites in patients with SLE and mild and critical IgA nephropathy compared to healthy controls
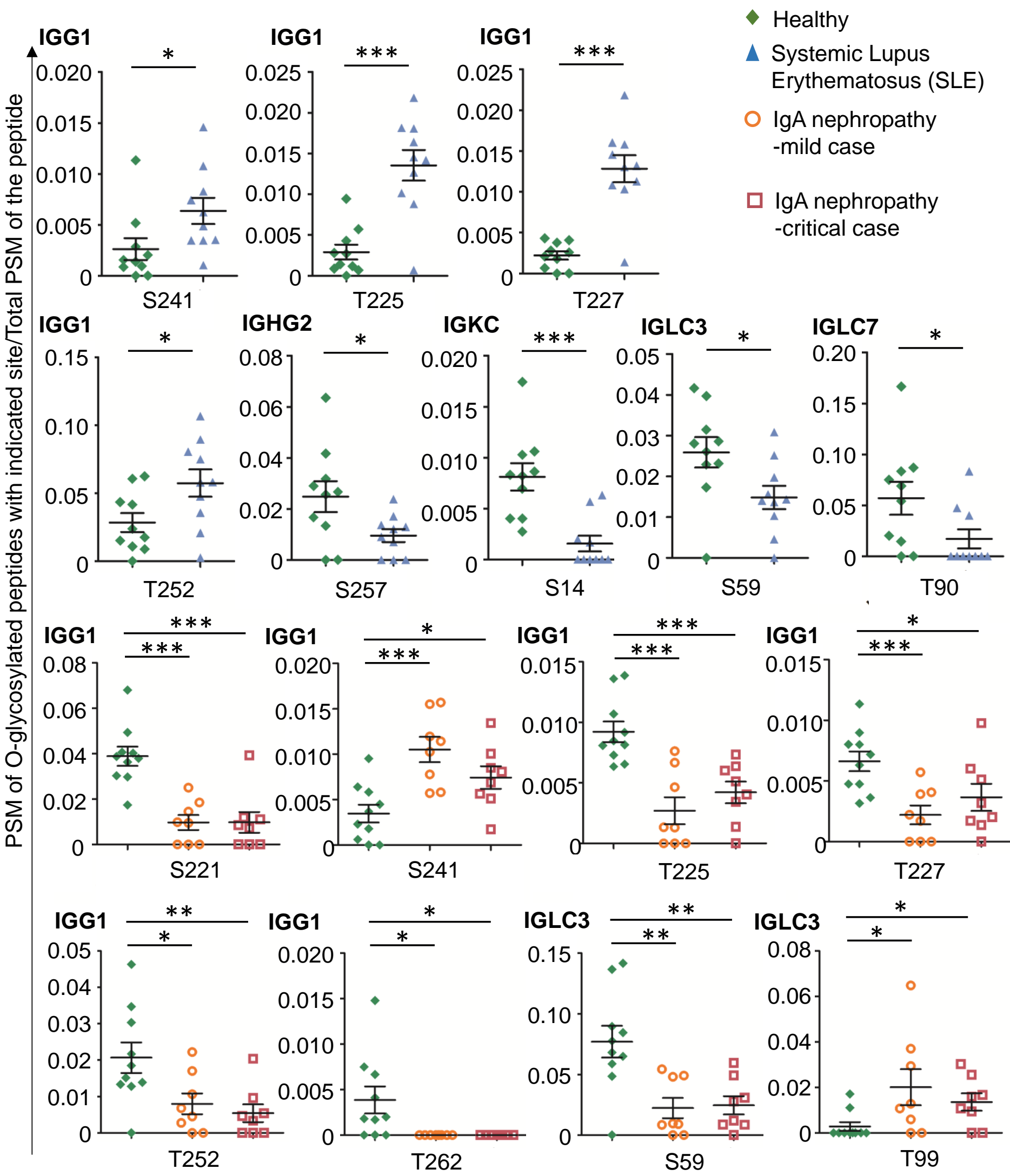
Table EV1 Numbers of O-glycoforms in the indicated regions of antibodies in human serum

\begin{tabular}{|c|c|c|c|c|c|c|c|c|c|c|c|c|c|c|c|}
\hline \multirow{3}{*}{ PTM } & \multirow{3}{*}{ M.W. } & \multicolumn{5}{|c|}{ Light chain } & \multicolumn{9}{|c|}{ Heavy chain } \\
\hline & & \multicolumn{4}{|c|}{ Variable region } & \multirow{2}{*}{$\begin{array}{l}\text { Constant } \\
\text { region }\end{array}$} & \multicolumn{4}{|c|}{ Variable region } & \multicolumn{5}{|c|}{ Constant region } \\
\hline & & CDR1 & CDR2 & CDR3 & $\mathrm{FR}$ & & CDR1 & CDR2 & CDR3 & FR & $\mathrm{CH} 1$ & $\mathrm{CH} 2$ & $\mathrm{CH} 3$ & $\mathrm{CH} 4$ & Hinge \\
\hline Fuc & 146.06 & 11 & 13 & 13 & 23 & 101 & 12 & 3 & & 59 & 14 & 28 & 11 & 7 & \\
\hline Man/Hex & 162.05 & 9 & 11 & 10 & 14 & 84 & 2 & & 2 & 22 & 14 & 42 & 16 & 9 & 11 \\
\hline HexNAc & 203.08 & & 1 & 6 & 9 & 49 & 2 & 2 & & 29 & 7 & 39 & 3 & 21 & 14 \\
\hline HexFuc & 308.11 & 1 & & 2 & 2 & 58 & 6 & 1 & & 32 & 10 & 2 & 8 & & 1 \\
\hline HexNAcFuc & 349.14 & & 1 & 1 & 2 & 19 & & & & 1 & & 1 & & 3 & \\
\hline HexHexNAc & 365.13 & & 2 & 9 & 6 & 17 & & 1 & 1 & 1 & 7 & 1 & 6 & 18 & 28 \\
\hline$(\mathrm{HexNAc})_{2}$ & 406.16 & 2 & 1 & 10 & 1 & 21 & 2 & & & 8 & 8 & 13 & 8 & 14 & 8 \\
\hline NeuAcFuc & 437.15 & & 4 & 6 & 1 & 44 & 1 & & & 23 & 7 & & 7 & 4 & 5 \\
\hline HexNeuAc & 453.15 & & & 2 & 2 & 14 & & & & 11 & 2 & 2 & 9 & 4 & 2 \\
\hline HexNAcNeuAc & 494.17 & & & 4 & 1 & 21 & 4 & & & 5 & 2 & 1 & 1 & 2 & 3 \\
\hline HexHexNAcFuc & 511.19 & & 2 & 2 & 6 & 27 & 1 & & & 10 & 8 & 3 & 7 & 7 & 7 \\
\hline $\mathrm{Hex}(\mathrm{HexNAc})_{2}$ & 568.21 & & & 6 & & 11 & & & & 12 & 7 & 3 & 4 & 13 & 36 \\
\hline 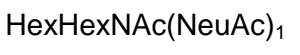 & 656.23 & & & & 1 & 4 & & & & & & 3 & & & 2 \\
\hline 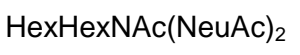 & 947.32 & & & 4 & & 6 & & & 1 & 1 & 4 & 2 & 6 & 2 & 2 \\
\hline
\end{tabular}


Table EV2 Numbers of O-glycosites in the indicated regions of different antibody isotypes

\begin{tabular}{|c|c|c|c|c|c|c|c|c|c|}
\hline \multirow{2}{*}{ Region } & \multicolumn{4}{|c|}{$\lg G$} & \multicolumn{2}{|c|}{$\lg A$} & \multirow{2}{*}{$\lg D$} & \multirow{2}{*}{$\lg M$} & \multirow{2}{*}{$\lg \mathrm{E}$} \\
\hline & $\lg G 1$ & $\lg G 2$ & $\lg G 3$ & $\lg G 4$ & $\lg A 1$ & $\lg A 2$ & & & \\
\hline$\overline{\mathrm{FR}}$ & 2 & & & & & 18 & 4 & 3 & \\
\hline CDR1 & 9 & & & & & & & & \\
\hline CDR3 & & & & & & & 3 & 1 & \\
\hline $\mathrm{CH} 1$ & 8 & 3 & 1 & 4 & 3 & & 3 & 66 & \\
\hline $\mathrm{CH} 2$ & 35 & 13 & 14 & 15 & 1 & 11 & 1 & 41 & \\
\hline $\mathrm{CH} 3$ & 18 & 13 & 6 & 11 & 19 & 4 & 1 & 15 & 7 \\
\hline $\mathrm{CH} 4$ & & & & & & & & 101 & \\
\hline Hinge & 5 & & 3 & & 110 & & 1 & & \\
\hline O-glycosites & 77 & 29 & 24 & 30 & 133 & 33 & 13 & 227 & 7 \\
\hline O-glycopeptides & 58 & 23 & 20 & 20 & 71 & 28 & 10 & 145 & 5 \\
\hline
\end{tabular}


Table EV3 Characteristics of the disease and control cohorts See excel form named "Table EV3"

Table EV4 Raw data of the O-glycosylations including total PSM, total O-glycopeptide PSM of the O-glycopeptides mentioned in the study

See excel form named "Table EV4" 